\title{
Numerical Experiments with a Turbulent Single-Mode Rayleigh-Taylor Instability
}

\author{
L.D. Cloutman
}

April 1, 2000

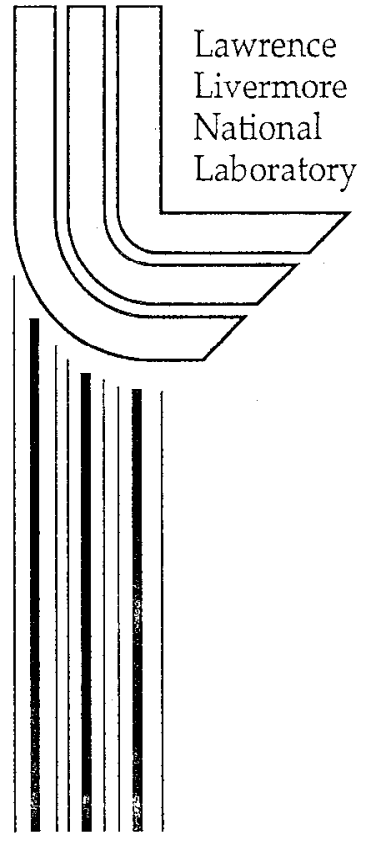


Lawrence Livermore National Laboratory

April 2000

\title{
NUMERICAL EXPERIMENTS WITH A TURBULENT SINGLE-MODE RAYLEIGH-TAYLOR INSTABILITY
}

\author{
Lawrence D. Cloutman \\ Lawrence Livermore National Laboratory \\ Tivermore, California 94550
}

\begin{abstract}
Direct numerical simulation is a powerful tool for studying turbulent flows. Unfortunately, it is also computationally expensive and often beyond the reach of the largest, fastest computers. Consequently, a variety of turbulence models have been devised to allow tractable and affordable simulations of averaged flow fields. Unfortunately, these present a variety of practical difficulties, including the incorporation of varying degrees of empiricism and phenomenology, which leads to a lack of universality. This unsatisfactory state of affairs has led to the speculation that one can avoid the expense and bother of using a turbulence model by relying on the grid and numerical diffusion of the computational fluid dynamics algorithm to introduce a spectral cutoff on the flow field and to provide dissipation at the grid scale, thereby mimicking two main effects of a large eddy simulation model. This paper shows numerical examples of a single-mode Rayleigh-Taylor instability in which this procedure produces questionable results. We then show a dramatic improvement when two simple subgrid-scale models are employed. This study also illustrates the extreme sensitivity to initial conditions that is a common feature of turbulent flows.
\end{abstract}




\section{Introduction}

Direct numcrical simulation (DNS) is a very powerful but computationally challenging technique for studying turbulent flows. A true DNS, by my definition, is a computational fluid dynamics (CFD) simulation of a turbulent fluid flow in which the mesh is sufficiently refined to resolve all scales of the flow, down to the microscale. In this case, there is no need to introduce turbulence models because a turbulent flow, if one accepts the Navier-Stokes equations as an adequate approximation, is nothing but a very complex, transient laminar viscous flow. Unfortunately, the Kolmogorov microscale of a fully-developed turbulent flow is approximately $\mathrm{Re}^{3 / 4}$ times the integral scale. Thus, for $\mathrm{Re}=10^{4}$, a fairly low value, the integral scale is 1000 times the microscale. Since real turbulence is intrinsically threedimensional, a DNS would require a minimum of 1000 zones in each of three directions. Such

a calculation is at the extreme edge of what can be done with heroic efforts using optimized codes, simple gas physics, and weeks of time on the largest parallel computers [1].

Turbulence models were developed to allow us to simulate the gross behavior of turbulent flows by averaging out or filtering the high frequency components of the flow. One of the earliest of these models specifically aimed at practical CFD research is by Harlow and Nakayama [2], which began development of the now familiar $k-\epsilon$ model. A somewhat similar approach is the large eddy simulation (LES). Smagorinsky [3] published an algebraic version for meteorological use, and this concept was combined with the filter function approach to provide a foundation for more modern LES models. It is beyond the scope of this paper to review the various models now found in the CFD literature. We merely note that in general these models lead to mean flow equations that have the same form as the original NavierStokes equations plus some extra terms for turbulent fluxes (which are often approximated in terms of an eddy viscosity). Extra algebraic or partial differential equations are introduced to allow evaluation of the turbulence terms. In addition to increasing the complexity and running times of CFD codes, these models are not always very accurate. Because they contain a certain amount of phenomenology, empiricism, and simple dimensional analysis to effect closure of the equation set, they lack universality.

This situation has led some researchers to hypothesize that one can avoid the expense 
and bother of using a turbulence model by relying on the numerical method used in the CFD program to introduce the two major effects of a real turbulence model in cases where the resolution is inadequate to resolve all scales of motion. First, the grid (or the finite number of basis functions in a spectral technique) imposes a high-wavenumber cutoff on the turbulence energy spectrum. Second, numerical diffusion provides a source of dissipation for the kinetic energy that cascades to the highest resolved wavenumbers. Indeed, it is easy to show that the numerical diffusion of first-order donor cell (upwind) differencing is the same order of magnitude as the Smagorinsky eddy viscosity, which has led to the suggestion of using donor cell differencing as a "poor man's LES model." Unfortunately, the term DNS often has been misapplied to this practice, creating some unnecessary confusion, and as we shall see, also perhaps some erroneous conclusions.

There is a second practice that also has created some confusion: The term DNS has been used to refer to well-resolved two-dimensional calculations (for example, the combustion studies of Chen and collaborators [4]). One can argue that two-dimensional turbulence is of legitimate academic interest, and therefore the term "two-dimensional DNS" is meaningful. However, physically realizable turbulence is three-dimensional and has different cascade properties. Even so, such two-dimensional studies can be very useful and may produce physical insight provided that one exercises caution in interpreting the results. Indeed, the present study relies mainly on two-dimensional simulations, although no claim to have performed a DNS is made.

In this report, we consider a classical Rayleigh-Taylor instability simulated with the COYOTE computer program [5]. COYOTE solves the compressible multicomponent NavierStokes equations for both cold and reactive flows in two-dimensional Cartesian and axisymmetric cylindrical geometries. It includes the LUVD11 turbulence model $[6,7]$ and the Smagorinsky model $[3,8]$ as options. The former subgrid-scale (SGS) turbulence model employs a single transport equation for the turbulence kinetic energy density. The latter model employs an algebraic SGS eddy viscosity.

The Rayleigh-Taylor problem is described in Section 2, along with estimates of some relevant parameters. Section 3 describes 12 numerical simulations performed at three different levels of resolution, each by four different numerical options. Also presented is a brief 
discussion of the instability growth rate. Section 4 presents additional solutions that provide additional insight. Section 5 presents the summary and conclusions.

\section{The Rayleigh-Taylor Instability}

In the classical Rayleigh-Taylor instability, a layer of dense fluid is placed on top of a layer of less dense fluid. Buoyancy forces cause an initial perturbation of the interface to grow, allowing the denser fluid to fall downwards, displacing the lighter fluid upwards, at an increasing rate. Eventually, for immiscible fluids, the system ends up at rest with the denser fluid on the bottom, and the lighter fluid on the top. For miscible fluids such as the gases considered here, some mixing at the molecular level will occur. In this example, the flow becomes turbulent, which enhances the molecular mixing. The final state will be a stratified mixture whose composition profile will depend on the details of the flow.

The situation considered in this report has an interface perturbed by a single Fourier mode as shown in Figure 1. Gas with a density of $1.57 \times 10^{-2} \mathrm{~g} / \mathrm{cm}^{3}$ lies above a layer of gas with a density of $1.0 \times 10^{-2} \mathrm{~g} / \mathrm{cm}^{3}$, which gives an Atwood number of At $=0.222$. The temperature is a uniform $287.7 \mathrm{~K}$. Both fluids are perfect gases with $\gamma=5 / 3$ and with molecular weights of 15.7 and 10.0 for the heavy and light gases respectively. A gravitational acceleration of $6.86 \times 10^{4} \mathrm{~cm} / \mathrm{s}^{2}(70 \mathrm{G})$ points downward. Rigid free-slip boundaries are used on all four walls. Transport coefficients are computed from the Lennard-Jones model [9] using some arbitrary but typical values for the potential parameters: $\sigma=2.576$ and $3.621 \AA$ and $\epsilon / k=10.20$ and 97.53 for species 1 (heavy) and 2 (light), respectively.

The dimensionless parameters for this problem are estimated from the solution at $40 \mathrm{~ms}$ when the instability is well developed but has not been disrupted by the heavy fluid hitting the bottom of the grid. Adiabatic sound speeds are $5.0 \times 10^{4} \mathrm{~cm} / \mathrm{s}$ and $6.3 \times 10^{4} \mathrm{~cm} / \mathrm{s}$ in the heavy and light gases respectively. The highest speeds obtained at any time are a little over $600 \mathrm{~cm} / \mathrm{s}$, so the maximum Mach number is on the order of 0.01 . There is no unique Reynolds number associated with this problem as the velocity changes dramatically with time. However, using the maximum speed at $40 \mathrm{~ms}$ (approximately $300 \mathrm{~cm} / \mathrm{s}$ ), the viscosity of the less viscous gas $\left(0.01 \mathrm{~cm}^{2} / \mathrm{s}\right)$, and the $7.3 \mathrm{~cm}$ initial perturbation wavelength for the length scale, the Reynolds number is $2.2 \times 10^{4}$. If we choose the higher viscosity 
$\left(0.024 \mathrm{~cm}^{2} / \mathrm{s}\right)$, the Reynolds number at $40 \mathrm{~ms}$ is $10^{4}$, which still indicates that a turbulent flow should develop from the initially laminar instability. It would take a grid of one or two thousand zones on a side to perform a DNS.

Linear theory predicts the growth rate of an inviscid, incompressible Rayleigh-Taylor instability to be

$$
\eta=(\mathrm{At} k g)^{1 / 2},
$$

where At is the Atwood number, $k$ is the wavenumber of the perturbation, and $g$ is the acceleration of gravity. For our present problem, $\eta=114.4 \mathrm{~s}^{1}$, which implies the amplitude of our calculations at $40 \mathrm{~ms}$ would be

$$
h=h_{0} \exp (\eta t)=19.43 \mathrm{~cm} .
$$

Since our grid is only $8.8 \mathrm{~cm}$ tall, we are clearly either well into the nonlinear regime or into a situation where boundary interactions are important, if not both.

\section{The 12 Basic Solutions}

COYOTE was used with four different combinations of numerical method and turbulence model to produce planar two-dimensional solutions at three different levels of resolution. First, calculations were done with first order donor cell (upwind) differencing of the advection terms and no turbulence model. Second, calculations were done with the second order tensor viscosity (TV) method [10] and no turbulence model. Next, solutions with TV and the Smagorinsky model were performed. Finally, solutions with TV and the LUVD11 oneequation turbulence transport model $[6,7]$ were performed. For each method, the 7.3 by 8.8 $\mathrm{cm}$ mesh was covered by a uniform grid of $73 \times 88,146 \times 196$, and $292 \times 352$ zones. The highest resolution uscd is still a factor of at least 3 coarscr than requircd for a true DNS. Figures 2 through 13 show mass fraction contours for species 1 , the heavy gas, at a time of $40 \mathrm{~ms}$ for each of these 12 cases. Table 1 lists the COYOTE input file for the highest resolution LUVD11 run. 


\subsection{Donor Cell Solutions}

Figures 2 through 4 show the donor cell solutions at increasing resolution. The bubbles and spikes are almost symmetric about the midplane of the mesh. The initial interface, which was one zone wide, has been broadened significantly by numerical diffusion, but it still exhibits the expected vortex pair shed by the tip of the spike. Donor cell differencing has a numerical diffusivity of approximately

$$
\nu_{i, \mathrm{dc}}=0.5\left|u_{i}\right| \delta x_{i}\left(1-\frac{\left|u_{i}\right| \delta t}{\delta x_{i}}\right)
$$

in the $x_{i}$ direction [11]. Since the iterative algorithm that makes COYOTE partially implicit begins to become inefficient when acoustic waves can travel more than three to five zones in one time step, this problem was run with the factor in parentheses greater than about 0.97 . Therefore, we shall neglect it, and we estimate the numerical viscosity on the finest grid to be $4.4 \mathrm{~cm}^{2} / \mathrm{s}$ in the fastest part of the flow. This is over two orders of magnitude larger than the physical viscosity, and it is sufficient to make the advection algorithm monotonic. The effective Reynolds number of the simulation is a few hundred, and we have a solution that appears to be laminar.

Since the three figures differ successively by a factor of two in resolution, the numerical diffusivity decreases by a factor of two from one figure to the next. This decrease appears as the narrowing of the band of contours representing the interface, by the enhanced detail in the vortex pair, and by the increasing level of asymmetry between the spike and bubbles. The maximum velocity is 293,329 , and $349 \mathrm{~cm} / \mathrm{s}$ in figures 2 through 4 , respectively. The montonic nature of the advection is demonstrated by the observation that temperature fluctuations divided by the initial temperature are on the order of $10^{-4}$, which is the square of the Mach number, just as expected. 


\subsection{Tensor Viscosity Solutions}

Figures 5 through 7 were calculated with the TV method and no turbulence model. The numerical diffusivity is tensorial in nature, ${ }^{1}$

$$
\overrightarrow{\vec{\nu}}_{\mathrm{tv}}=0.5 \delta t \mathbf{u u}
$$

and much of this positive diffusivity is used to cancel diffusive truncation errors with negative diffusivities. At the location of the peak fluid speed, the numerical viscosity is approximately $0.04 \mathrm{~cm}^{2} / \mathrm{s}$ for the finest resolution case, and it will be lower elsewhere on the grid. This is only a factor of 4 larger than the lowest physical viscosity, so we have the physical viscosity comparable to the numerical viscosity over much of the grid where the flow speed is less than about $200 \mathrm{~cm} / \mathrm{s}$. As expected, the lowered numerical diffusion produces less broadening of the interface and allows much more detail to be present at the grid scale becanse the viscons diffusion time for the width of one zone is $\tau=\delta x^{2} / \nu=62.5 \mathrm{~ms}$, which is much larger than the dynamical timescale of $0.1 \mathrm{~ms}$ or so of the smallest resolved eddies. These three figures are the closest of any presented in this report to a typical pseudo-DNS computed with modern high order techniques, and they suffer from the same difficulties.

We note that these three figures exhibit a definite lack of grid independence. Figure 5 is the only one in this entire report that hits the bottom of the mesh in 40 ms. Figure 6 bears some resemblance to the donor cell solutions, but with a lot of fine structure along the interface. In Figure 7, the tip of the spike and bubbles are much flatter and exhibit strong secondary instabilitics. Maximum flow vclocitics are 463, 354, and $408 \mathrm{~cm} / \mathrm{s}$ for figures 5 through 7 , respectively.

These plots are exhibit a lot of fine structure. This detail is due to at least two sources. First, the flow has become turbulent, and these small structures are a manifestation of the chaotic nature of turbulent eddies. Second, a lot of this is just numerical "junk," almost certainly driven by dispersive truncation errors. As the grid is refined, the junk just gets finer. If we were running an Euler code, this would go on indefinitely, and it is possible that the large scales would be affected unphysically. We certainly see the secondary instabilities

\footnotetext{
${ }^{1}$ The donor cell diffusivity may also be written in matrix form, with diagonal elements given by equation 3 and with zeroes off the diagonal [10].
} 
becoming significant in some calculations based on the Euler equations [12]. In a DNS, viscosity, thermal conduction, and mass diffusion will eventually damp the small structures at the microscale, both the physical chaos and the numerical dispersion errors. One of the roles of a turbulence model is to damp the small-scale junk, usually by use of an eddy viscosity to introduce a realistic estimate of the dissipation rate. The value of the eddy viscosity needed to provide physically reasonable rates of dissipation may be very different than the numerical viscosity of the method. For TV, the eddy viscosity is larger; for donor cell, it can be smaller, as we shall now see.

\subsection{Smagorinsky Model Solutions}

Figures 8 through 10 show the solution for the TV method plus the Smagorinsky model with a model constant of 0.176 and a turbulence length scale of $1.94 \delta x$. Broadening of the interface is approximately half that of donor cell differencing at the same resolution, and some of the larger eddies along the interface are visible. The "junk" has been brought under control since the Smagorinsky model has the property that it expands the microscale up to the grid scale [13]. The secondary instability at the tip of the spike is still quite noticeable, even at the coarsest resolution. Additional secondary vortices appear as the resolution is improved. As one would expect, there is no grid independence since the resolution is still well below the microscale even in Figure 10.

The question naturally arises as to whether the secondary vortices are physical or a numerical artifact of the dispersive truncation errors. Since the locations of some of them are nearly the same in going from figure 9 to figure 10, they could well be physical. Also, there is the beginning of a secondary vortex at the tops of the bubbles in figure 4 corresponding to a strong vortex in both figures 9 and 10. The few clear photographs of turbulent RayleighTaylor instabilities that I was able to find fail to yield an unambiguous example, but one of the most suggestive was figure 2 of reference [14]. However, a qualitatively similar feature is seen in the numerical simulation of thermals by Grabowski and Clark [15]. They argue that this is a physical result of a combination of baroclinic torques and shear. The same effects are present in the Rayleigh-Taylor simulations.

The peak velocities in figure 8 through 10 are 324,386 , and $387 \mathrm{~cm} / \mathrm{s}$, respectively. 
The peak eddy viscosities are $2.51,1.07$, and $0.40 \mathrm{~cm} / \mathrm{s}^{2}$, which are well above the numerical and molecular viscosities.

\subsection{LUVD11 Model Solutions}

Figures 11 through 13 show the solution for the TV method plus the LUVD11 turbulence model. LUVD11 is constructed so that for turbulence with equilibrium between production and decay, one obtains the same eddy viscosity as the Smagorinsky model. The same turbulence length scale $(1.94 \delta x)$ is used in both calculations. However, the LUVD11 model produces more smoothing than the Smagorinsky model. At the lowest resolution, the interface thickness is almost as large as for donor cell differencing, and the vortex formation is even more suppressed. At the finest resolution, the interface is beginning to show some structure, but not nearly as much as the Smagorinsky model.

The peak velocities in figure 11 through 13 are 296, 347, and $391 \mathrm{~cm} / \mathrm{s}$, respectively. The peak eddy viscosities are $3.10,1.63$, and $0.88 \mathrm{~cm} / \mathrm{s}^{2}$, which is somewhat larger than the Smagorinsky eddy viscosities.

There are two important differences between the two turbulence models: First, the LUVD11 does not assume the turbulence production rate equals the decay rate. Second, the LUVD11 model allows for advection and self-diffusion of turbulence. While both of these factors favor the use of the LUVD11 model, resolution of the differences between these two models will require additional research.

\subsection{Instability Growth Rate}

It is interesting that with the exception of Figure 5, these calculations all predict pretty much the same amplitude of the instability regardless of the fine details. This insensitivity of the thickness of the mixed layer to details of numerical method and turbulence model suggests that the growth rate of Rayleigh-Taylor instabilities may not be a good diagnostic for testing turbulence models.

Figure 14 shows a plot of bubble and spike amplitudes versus time for the highresolution donor cell solution, figure 4. Amplitudes were taken as the highest and lowest locations of the 0.5 mass fraction contour. At early times, the numerical growth lags well 
behind the theoretical exponential growth of the linear regime. At late times, the amplitude is expected to scale as $\alpha \mathrm{At} g t^{2}$. However, visual examination of the plot fails to show a $t^{2}$ segment in the curves. Plotting the curves against $t^{2}$ instead of $t$ also shows no clear sign of a $t^{2}$ law. On the contrary, at later times the curves grow linearly in time before decelerating due to boundary interactions. Clearly, the conditions of these numerical experiments are too restrictive to allow the development of an unambiguous $t^{2}$-scaling regime. Attempts to fit the points just to the left of the linear section ( $25 \mathrm{~ms}$ and earlier) with a parabola led to large values of $\alpha$ (0.09 and larger) for a very brief time. In the nomenclature of Linden, et al. [14], our simulations employ $a_{0} / \lambda=0.027$. Their Table 1 shows that $\alpha=0.09$ is only a little larger than expected if we had indeed produced a $t^{2}$ regime. This result is probably fortuitous.

\section{Miscellaneous Numerical Experiments}

Four additional calculations were performed to test various aspects of the discussion in the previous section. First, the previous calculations were all symmetric about the vertical midplane. Figure 6, the medium resolution TV run with no turbulence model was rerun with a small perturbation that breaks the symmetry. Second, finite difference codes can have bounded solutions that exhibit the same type of chaos as iterated nonlinear maps [16]. This same case was rerun with smaller time steps to make sure the fine structure was not duc to this type of chaos. Third, the LUVD11 case was run with the turbulence length scale set to $3.75 \delta x$ as recommended in [7] rather than $1.94 \delta x$ as used in figures 11 through 13. Finally, the coarse-grid Smagorinsky case was run in three dimensions with a small perturbation to break the symmetry in all directions.

\subsection{Broken Symmetry}

Figure 15 shows the effect of a tiny perturbation to the initial condition used to generate figure 6. A four-zone block centered at $x=5.5 \mathrm{~cm}$ and $y=4.45 \mathrm{~cm}$ was filled with heavy material instead of light, and the temperature was increased from $287.7 \mathrm{~K}$ to $290.0 \mathrm{~K}$. As shown in figure 1 , this perturbation is about three quarters of the way to the right and slightly below the interface. In addition to the symmetry of the density field being broken, the small 
temperature increase produces a pressure increase that drives a weak circular acoustic wave that creates a slight asymmetry in the velocity field. What we see in figure 15 compared to figure 6 is that the flow in the left side of the grid is affected only the slightest amount, but many details are different on the right hand side, even a considerable distance from the initial four-zone perturbation. Such sensitivity is a feature of the mathematical chaos that can occur in solutions of nonlincar partial differential equations and nicely illustrates the predictability problem (the butterfly effect) for turbulent flows.

Figure 16 shows the mass fraction plot of the material in the initial four-zone symmetrybreaking perturbation. Its peak mass fraction has decreased from unity to 0.048 due to a combination of molecular and numerical diffusion. It is displaced from its initial position and has been significantly stretched by convection. It is essential to understand this phenomenon in order to understand how inhomogenities become mixed at the atomic level by turbulence.

\subsection{Another Type of Chaos?}

There is a second type of chaos that can occur in finite difference CFD simulations, and it may occur also in finitc element methods as well. It is well known that nonlincar itcratcd maps, such as the logistic map, exhibit a discrete type of chaos under certain conditions, usually when a free parameter exceeds a critical value. What is less often recognized is that finite difference algorithms for CFD are nonlinear iterated maps, and therefore might exhibit this kind of chaos when the time step becomes too large. Note that this is different than the chaos in the continuous solutions of differential equations. This chaos appears as apparently random solutions of the finite difference equations. These are valid solutions of the difference equations, but they are a qualitatively different class of solution than the approximate numerical solutions to the underlying differential equations found in the limit of infinite spatial and temporal resolution. Such chaos has been observed in COYOTE [16] as well as other codes [17]-[20]. It occurs when the code is run near its stability limit. What we observe is that the codes have two critical time step limits. First, the one that is usually considered, leads to unbounded solutions for time steps larger than the familiar stability limit ("the code blows up") encountered in linear stability analysis. Second, more subtly, if the time step is below a smaller limiting value, the finite difference solution will 
be as smooth as the differential equation solution (assuming adequate resolution), and this smooth solution approaches the differential equation solution as the time step is reduced (providing the method is stable and consistent). If the second limit is smaller than the first, there will be a range of time steps in which the code will produce a bounded solution (that is, will not blow up), but the solution will contain "random junk" (actually, discrete deterministic chaos) that bears no resemblance to the solutions of the differential equations.

The test for the presence of this discrete chaos is simple: Rerun one of the junky solutions with a reduced time step. This was done for the medium resolution TV case shown in Figure 6. This run required 35062 instead of 23918 time steps to reach $40 \mathrm{~ms}$. No plot is shown because the mass fraction contours are identical except for a few very small features. The tiny perturbation used to make figure 15 made a much larger difference, and hence we rule out this discrete chaos as a factor in the present solutions.

\subsection{LUVD11 Length Scale Test}

An earlier paper [7] reported a set of parameters for use in the LUVD11 model based on the decay of a swirling flow. This parameter set also produced good results in an unpublished low-resolution simulation of a round turbulent jet. Both simulations were in two-dimensional cylindrical coordinates. The calculations shown in figures 17 through 19 used a length scale of $L=3.75 \delta x$ as recommended in [7] instead of the value $1.94 \delta x$ used to compute figures 11 through 13.

This larger value produced more smoothing in the Rayleigh-Taylor simulations since the eddy viscosity is expected to scale as $L^{2 / 3}$ if $L$ is in the inertial subrange. ${ }^{2}$ The peak velocities in figure 17 through 19 are 261,302, and $353 \mathrm{~cm} / \mathrm{s}$, respectively. The peak eddy viscosities are $5.77,2.92$, and $1.62 \mathrm{~cm} / \mathrm{s}^{2}$, which is roughly twice as large as the earlier LUVD11 eddy viscosities. Figure 18 strongly resembles figure 11, as expected. However, the shape of the spike looks odd in figure 17, with far too much diffusion. There are some differences between figures 12 and 19, with too much flattening of the tip of the spike in figure 19 .

\footnotetext{
${ }^{2}$ The eddy viscosity is proportional to $\delta x \mathcal{K}^{1 / 2}$, where $\mathcal{K}$ is the subgrid scale turbulence kinetic energy. If the resolution is in the $k^{-5 / 3}$ inertial subrange, $\mathcal{K}$ scales as $\delta x^{-2 / 3}$. The Smagorinsky eddy viscosity should scale the same way as the LUVD11 eddy viscosity.
} 
There are some significant differences between the present set of Rayleigh-Taylor simulations and the earlier successful validation problems. Both the swirling flow simulation and the jet used rather coarse zoning in two-dimensional cylindrical coordinates. Turbulent swirling flows have long been recognized as presenting special difficulties for two-equation turbulence models. In addition, the minimal LUVD11 model used in all calculations omits the Leonard and cross terms. The swirling flow simulation also omitted the buoyancy creation term, even though large stabilizing buoyancy forces are generated by the swirl (which may have been compensated by the large length scale). The swirling flow also has significant shear in the $\theta$ direction, which could easily break the axial symmetry of the flow via the Kelvin-Helmholtz instability. The swirling flow contained decaying fully-developed turbulence, whereas the Rayleigh-Taylor instability begins laminar and transitions to turbulence. The jet is more like the Rayleigh-Taylor instability in this regard. With all of these differences and omissions, the parameters of the LUVD11 model derived on the basis of such a limited set of validation problems must be considered highly uncertain and possibly lacking universality. Improvements to the model are under development and should improve the universality and predictive capability of the model.

There is an additional difficulty in determining the optimum parameter set. The earlier swirling flow and turbulent jet problems had no difficulties with dispersive truncation errors, but the present Rayleigh-Taylor simulations show them in the temperature field and, to a lesser degree, in the mass fractions. It is clear that a monotonic scheme must be implemented in COYOTE before the parameter set can be optimized with any hope of universality.

\subsection{A Three-Dimensional Case}

The three-dimensional version of COYOTE was used to repeat the coarse-grid case with the Smagorinsky model (figure 8). The grid in the third direction was 24 uniform $1.0 \mathrm{~mm}$ zones, which is a distance equal to approximately one third of the wavelength of the initial perturbation. A three-dimensional perturbation was imposed on the initial condition. It was located in the $(x, y)$ plane very close to that in the perturbed two-dimensional solution. In the other direction, it is 5 zones long and located off-center to create an asymmetric 
three-dimensional perturbation.

The $(x, y)$ cross sections of the solution are almost identical to figure 8 and hence are not shown. A slice in the $(y, z)$ plane at $x=5.0 \mathrm{~cm}$ is shown in figure 20 . At $40 \mathrm{~ms}$, the instability is still quite two-dimensional. We expect that the three-dimensional effects would get more pronounced at later times. This solution uses a coarse grid, and perhaps finer resolution would show something more dramatic. However, such a calculation is not cost-effective at this time, and we shall proceed on the assumption that three-dimensional effects will not seriously affect our conclusions.

This result is in contrast to Kane, et al. [12], who present numerical simulations of instabilities that exhibit a sensitivity to the dimensionality of the simulation. In their comparison, they chose a three-dimensional initial perturbation with the same total wavenumber

as in the two-dimensional case, but with $k_{x}=k_{y}=2^{-1 / 2} k$. The same linear growth rate will apply to both cases since it depends only on $k$, and not on the relative values of $k_{x}$ and $k_{y}$. We do not have any assurance that the same is true in the nonlinear regime, so their result, namely the three-dimensional instability grows somewhat faster, may not be relevant to the present study. This is an interesting open question for future work.

\section{Summary and Conclusions}

The primary objective of this study is to illustrate the effects of numerical methodology and turbulence modcls on a gencric Raylcigh-Taylor instability. This was accomplished with the COYOTE CFD program using four different numerical methods and turbulence models to produce numerical solutions at three different levels of resolution differing by factors of 2 . Four additional solutions were produced to address issues raised by the initial 12 runs. We came to the following conclusions:

- When simulating turbulent flows, the Euler equations are inappropriate for a true DNS where one must use resolution at the microscale because molecular transport processes play a fundamental role in the dynamics of turbulence. Indeed, the microscale itself is determined by the molecular transport and dissipation. If one solves the Euler equations, the numerical solution will contain features at the limit of resolution, even if that limit is much smaller than the physical cutoff. The impact of this unphysical bchavior on the 
larger scales is unknown and cannot be determined by the usual techniques, such as mesh refinement. The Navier-Stokes equations must be solved for a true DNS, and there is no point to computing the unphysical solutions at ever higher resolutions using the Euler equations. This obvious point seems to have been missed in some quarters.

- Even in simulations with resolution too coarse for a true DNS, the Euler equations are nol appropriate. The TV solutions in figures 5 through 7 show an example in which there is no clear convergence upon grid refinement. Indeed, the coarsest resolution produces a physically unrealistic solution that might look plansible to the untutored eye. Because the present numerical solutions show the sensitivity of many flow details to resolution, numerical methodology, and the choice of turbulence model, it is clear that results depend on the diffusivities, whether numerical, molecular, or eddy. It would be a matter of pure chance to find an accurate advection algorithm with just the right amount of numerical diffusion and dissipation to mimic the missing physical processes. Use of a turbulence model in conjunction with a low-diffusion advection method is the correct approach, using the eddy viscosity to set the proper level of dissipation. Using the Navier-Stokes equations with eddy diffusivities has the further advantage of retaining the Galilean and tensor invariance of the full governing equations.

- The insensitivity of the thickness of the mixed layer to details of numerical method and turbulence model suggests that the growth rate of Rayleigh-Taylor instabilities is not a good diagnostic for testing turbulence models.

- It is important to run a problem on at least two significantly different grids to test the adequacy of the resolution. For ensemble-averaged turbulence models, the ideal situation is to refine the mesh until a grid-independent solution is obtained. For LES models, the solution will never approach true grid-independence because of the construction of the model to resolve as much detail as the grid allows. Even so, the resolution must still be varied to make sure that at least the integral scale features are adequately simulated. Grid and time step variation is perhaps the best protection against specious results in cases where there is little experimental data or only global quantities have been measured.

- Numerical gimmicks such as higher order methods are no substitute for resolution. At a minimum, there must be a few zones across the width of each resolved flow feature. 
- The TV method by itself produced unacceptable results due to the disperive truncation errors, which are a side effect of the low numerical diffusion. This method would benefit from the incorporation of a monotonicity-preserving flux limiter. This is true even for cases using a turbulence model that has a substantial eddy viscosity.

- The low resolution solutions are all much the same. Big differences appear at higher resolutions, just where all stable and consistent methods should exhibit convergence and where the subgrid-scale models should become less important. At the resolution used in this study, methodology matters.

- The present problem appears to exhibit no significant difference between twodimensional and three-dimensional simulations when the same $x$ wavenumber is used and $k_{y}=\infty$. It is possible that the limited problem time and boundary effects influence this conclusion, making it problem-dependent.

- There is a need to reoptimize the LUVD11 parameters, as evidenced by the need to adjust the turbulence length scale from earlier recommended values. It is possible that this is due to the need to improve the transport equation in several areas: Inclusion of backscatter, inclusion of the Leonard terms, counter-gradient diffusion, and an improved buoyancy creation term head the list.

- The turbulence models used here were developed for fully developed turbulence. The present study simulates a problem that makes a transition to turbulence, going from an initially laminar flow to low intensity turbulence to fully developed turbulence. It may be necessary to modify the turbulence models to account for this behavior.

- The LUVD11 solution with the same length scale as the Smagorinsky model produced more smoothing than the latter, even though the minimal LUVD11 reduces to the Smagorinsky model for homogeneous, statistically steady turbulence. In the present problem, the turbulence is decidedly inhomogeneous and unsteady, so the self-diffusion of nonequilibrium turbulence kinetic energy provides might explain the difference. This is an example of algebraic and transport models showing significant differences.

- The high-resolution runs show pronounced secondary instabilities, although the details are dependent on how we did the grid-scale dissipation. It is an open question how physical these flow features are. Suggestively, the limited selection of available photographs 
of turbulent Rayleigh-Taylor instabilities sometimes show qualitatively similar fine structure.

\section{Acknowledgements}

It is a pleasure to acknowledge several useful conversations with John Ramshaw. This work was performed under the auspices of the U. S. Department of Energy and University of California Lawrence Livermore National Laboratory under contract No. W-7405-Eng-48.

\section{References}

[1] A. A. Mirin, R. H. Cohen, B. C. Curtis, W. P. Dannevik, A. M. Dimits, M. A. Duchaineau, D. E. Eliason, D. R. Schikore, S. E. Anderson, D. H. Porter, P. R. Woodward, L. J. Shich, and S. W. White, "Very High Resolution Simulation of Compressible Turbulence on the IBM-SP System," Lawrence Livermore National Laboratory report UCRL-JC-134237, 1999.

[2] F. H. Harlow and P. I. Nakayama, "Turbulence transport equations," Phys. Fluids 10, $2323(1967)$.

[3] J. Smagorinsky, "General circulation experiments with the primitive equations I. The basic experiment," Mon. Weather Rev. 91, 99 (1963).

[4] J. H. Chen, T. Echekki, and W. Kollmann, "The mechanism of two-dimensional pocket formation in lean premixed methane-air flames with implications to turbulent combustion," Combust. Flame 116, 15 (1999).

[5] Clontman, L. D., "COYOTE: A Computer Program for 2-D Reactive Flow Simulations," Lawrence Livermore National Laboratory report UCRL-ID-103611, 1990.

[6] Cloutman, L. D., "The LUVD11 Large Eddy Simulation Model," Lawrence Livermore National Laboratory report UCRL-ID-107128, 1991.

[7] L. D. Cloutman, "Numerical simulation of the heat transfer and decay of turbulent swirling flow," Comput. \& Fluids, 17, 437 (1989). 
[8] J. W. Deardorff, "On the magnitude of the subgrid scale eddy coefficient," J. Comput. Phys. 7, 120 (1971).

[9] L. D. Cloutman, "A Database of Selected Transport Coefficients for Combustion Studies," Lawrence Livermore National Laboratory report UCRL-ID-115050, 1993.

[10] J. K. Dukowicz and J. D. Ramshaw, "Tensor viscosity method for convection in numerical fluid dynamics," J. Comput. Phys. 32, 71 (1979).

[11] P. K. Smolarkiewicz, "A simple positive definite advection scheme with small implicit diffusion," Mon. Weather Rev. 111, 479 (1983).

[12] J. Kane, D. Arnett, B. A. Remington, S. G. Glendinning, G. Bázan, E. Müller, B. A. Fryxell, and R. Teyssier, "Two-dimensional versus three-dimensional supernova hydrodynamic instability growth," Ap. J. 528, 989 (2000).

[13] J. D. Ramshaw, "Alternative Interpretation of the Subgrid Scale Eddy Viscosity", Los Alamos Scientific Laboratory report LA-7955-MS, 1979.

[14] P. F. Linden, J. M. Redondo, and D. L. Youngs, "Molecular mixing in Rayleigh-Taylor instability," J. Fluid Mech. 265, 97 (1994).

[15] W. W. Grabowski and T. L. Clark, "Cloud-environment interface instability: Rising thermal calculations in two spatial dimensions," J. Atm. Sci. 48, 527 (1991).

[16] L. D. Cloutman, "Chaos and Instabilities in Finite Difference Approximations to Nonlinear Differential Equations," Lawrence Livermore National Laboratory report UCRLID-131333, 1998.

[17] H. C. Yee, P. K. Sweby, and D. F. Griffiths, "Dynamical approach study of spurious steady-state numerical solutions of nonlinear differential equations. I. The dynamics of time discrelization and its implications for algorithm development in compulational fluid dynamics," J. Comput. Phys. 97, 249 (1991). 
[18] H. C. Yee and P. K. Sweby, "The dynamics of some iterative implicit schemes," in Chaotic Numerics, Contemporary Mathematics 172, (P. E. Kloeden and K. J. Palmer, Eds.), American Mathematical Society, Providence, RI, 1994, pp. 75-96.

[19] H. C. Yee, J. R. Torczynski, S. A. Morton, M. R. Visbal, and P. K. Sweby, "On spurious behavior of CFD simulations," AIAA Paper 97-1869 (1997).

[20] H. C. Yee and P. K. Sweby, "Aspects of numerical uncertainties in time marching to steady-state numerical solutions," AIAA J. 36, 712 (1998). 
Table 1. COYOTE Input File for Rayleigh-Taylor Instability

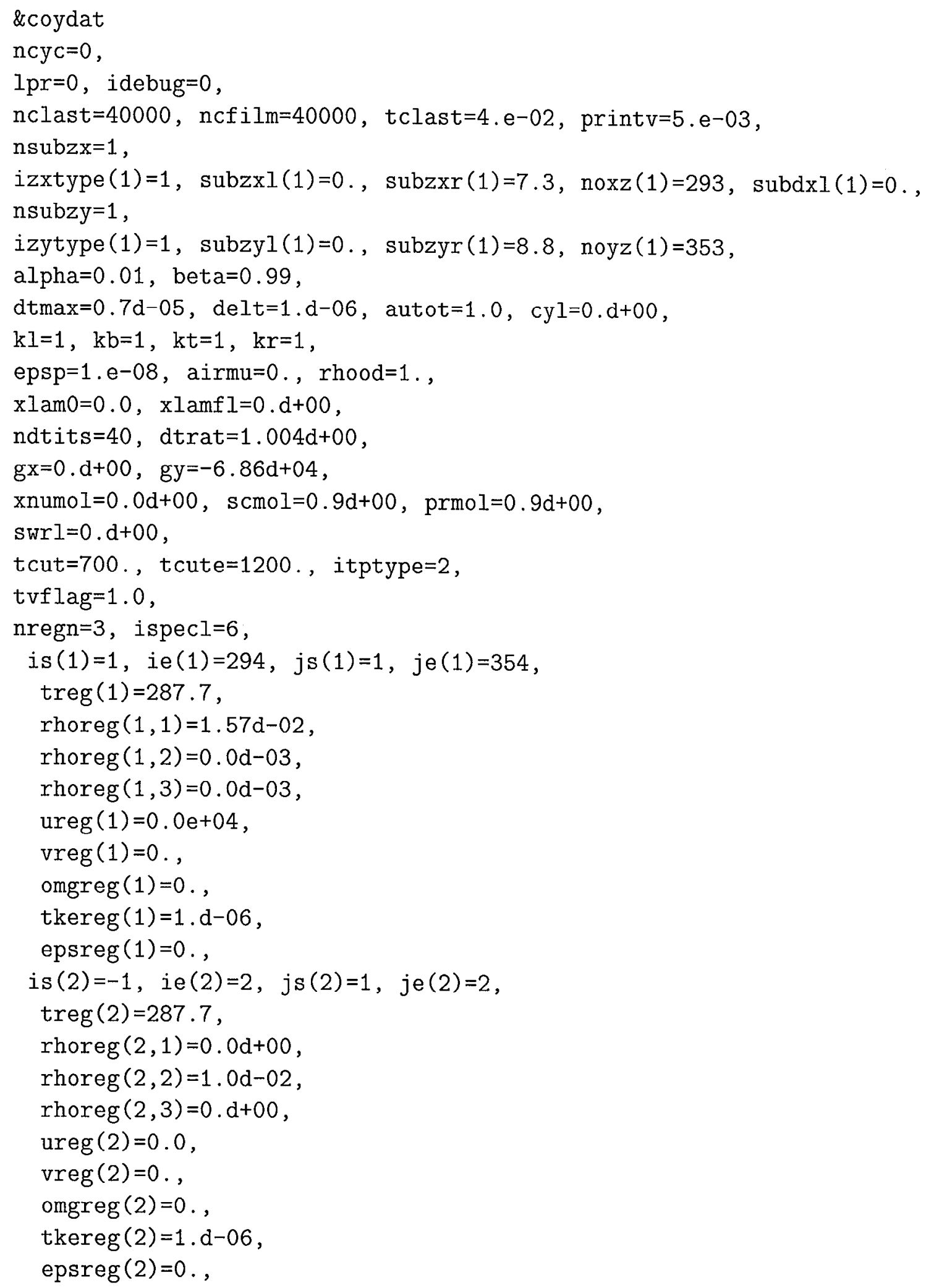




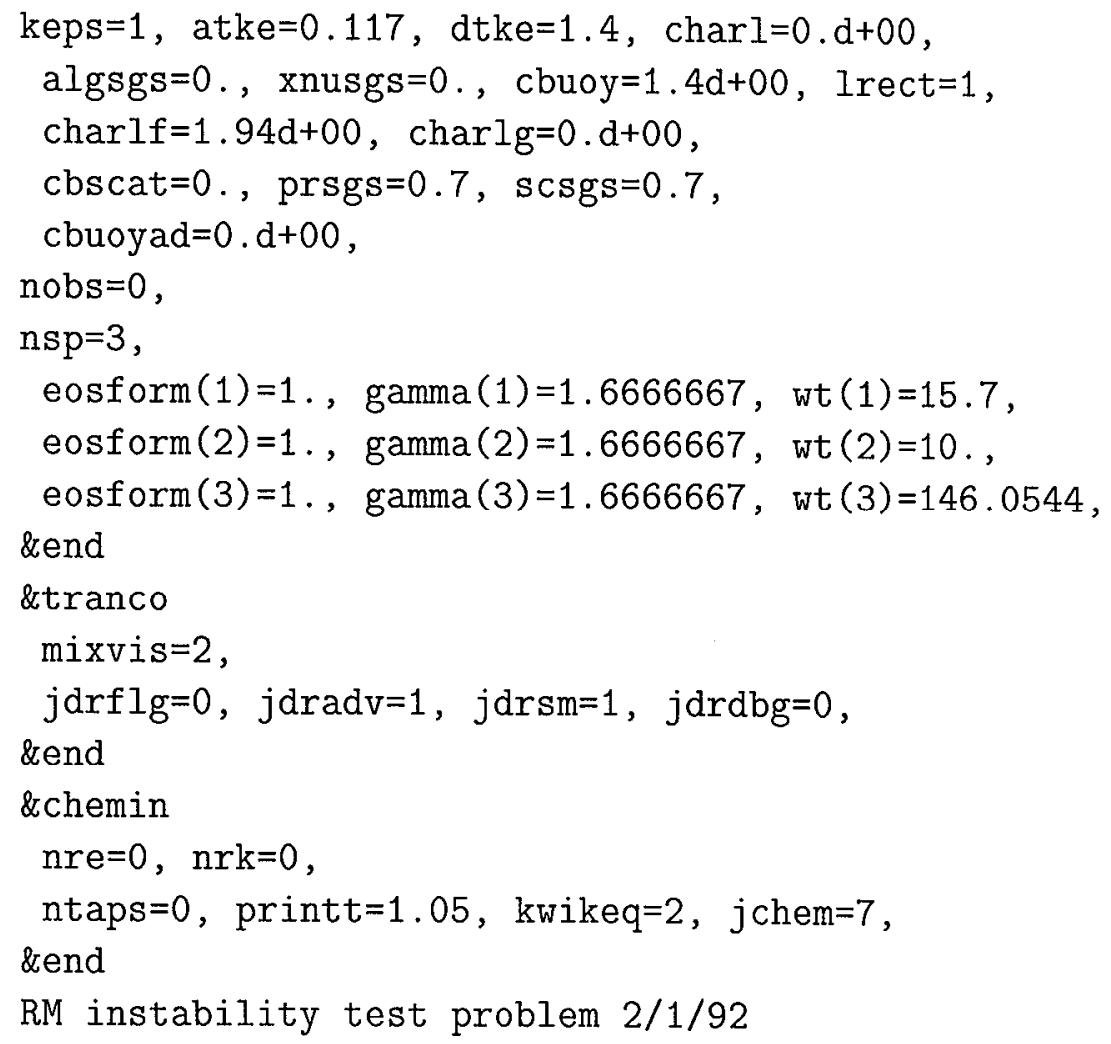

RM instability test problem 2/1/92 


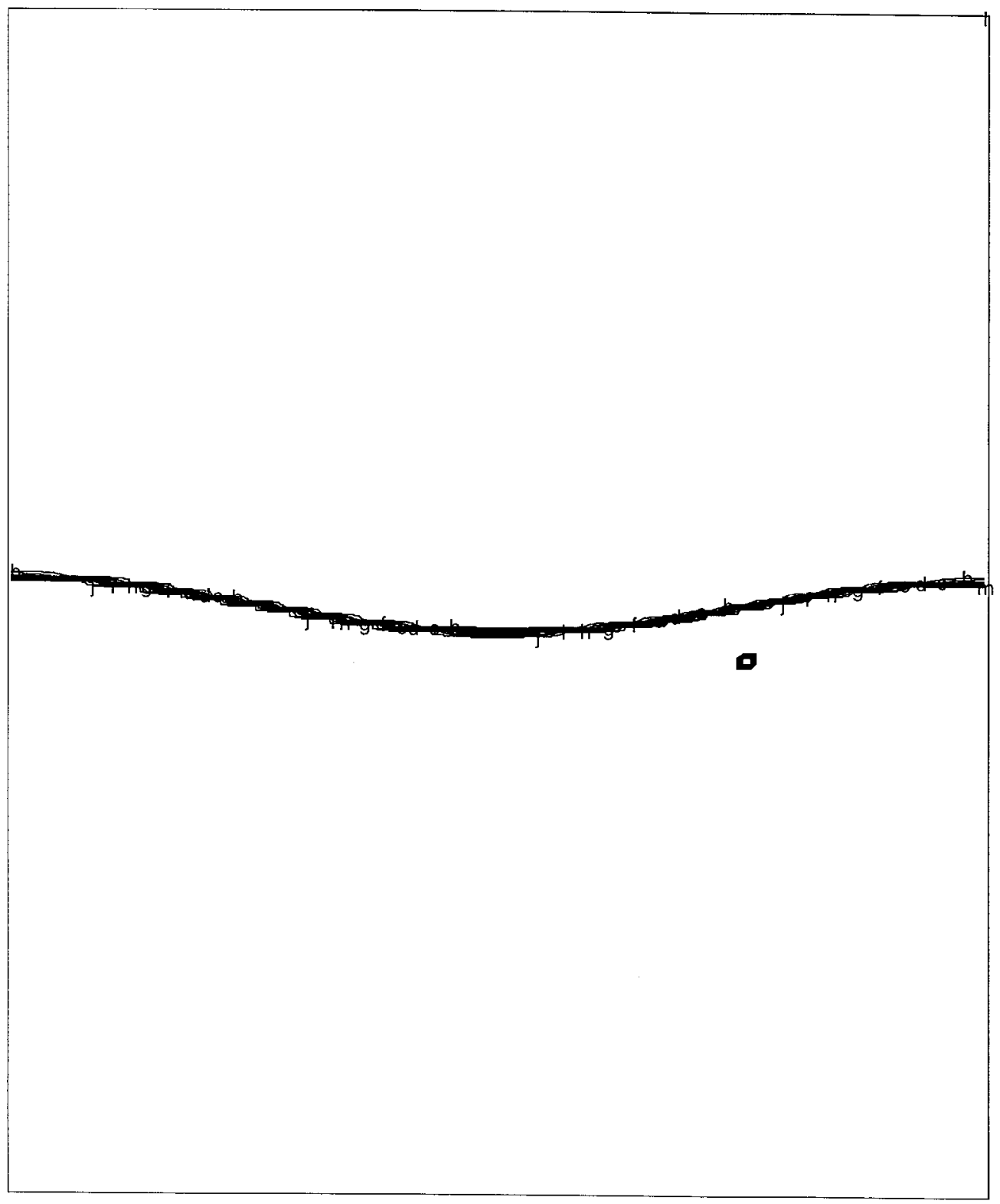

Figure 1: Mass fraction contours for species 2 at $0 \mathrm{~ms}$. The heavy gas (species 1 ) is on the top. The grid is $7.3 \mathrm{~cm}$ wide by $8.8 \mathrm{~cm}$ tall, and it is covered with $146 \times 196$ uniform zones. The initial interface is $y=4.4 \mid 0.2 \cos (2 \pi x / 7.3)$. In all plots, the contour labels $b$ through $j$ denote contour values of 0.1 through 0.9 in steps of 0.1 . The small square just below the interface is a perturbation described in the text that is used in only one of the simulations, Figures 15 and 16. 


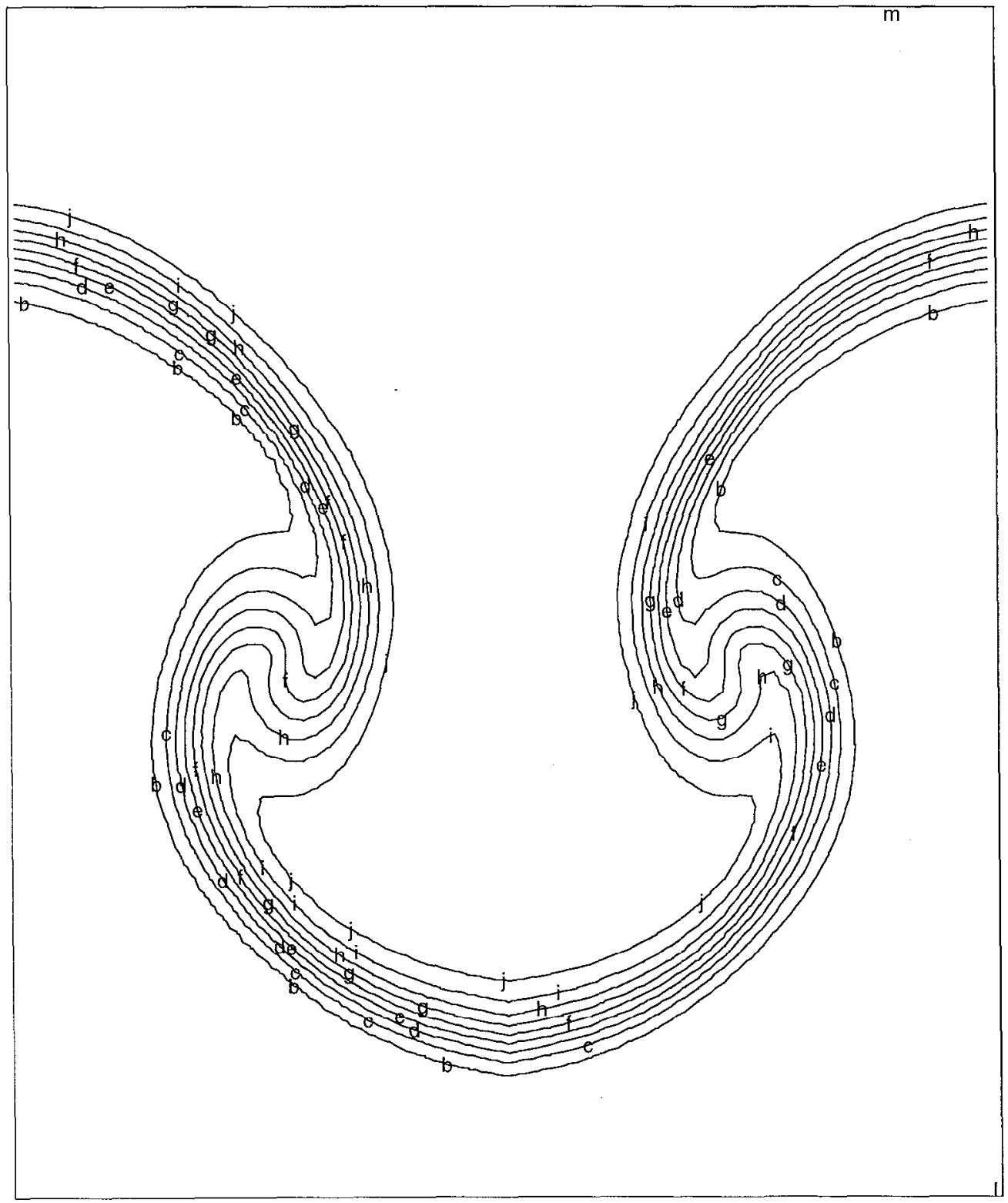

Figure 2: Mass fraction contours for species 1 at $40 \mathrm{~ms}$. The grid is $73 \times 88$, and the calculation is done with donor cell differencing and no turbulence model. 


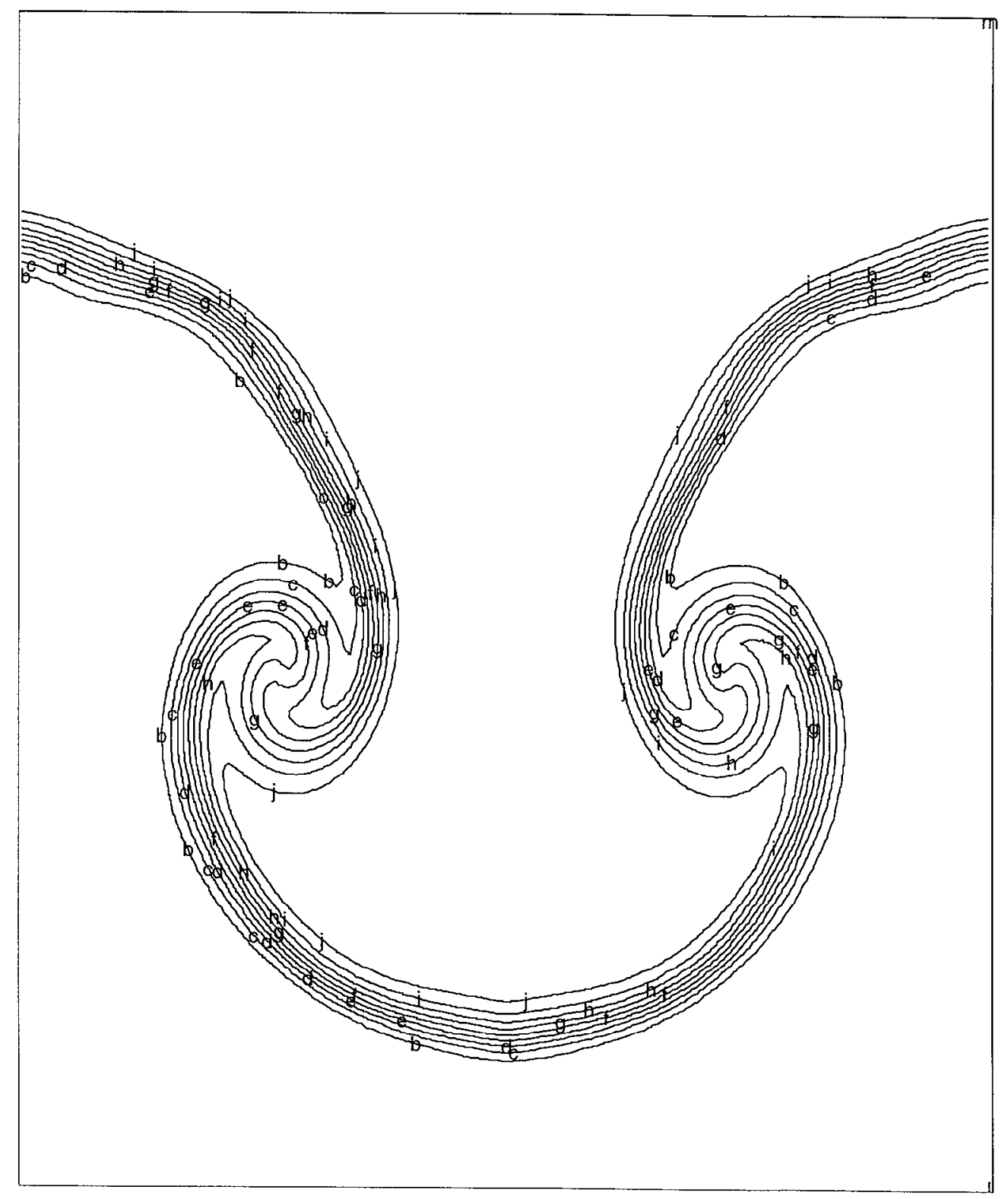

Figure 3: Mass fraction contours for species 1 at $40 \mathrm{~ms}$. The grid is $146 \times 196$, and the calculation is done with donor cell differencing and no turbulence model. 


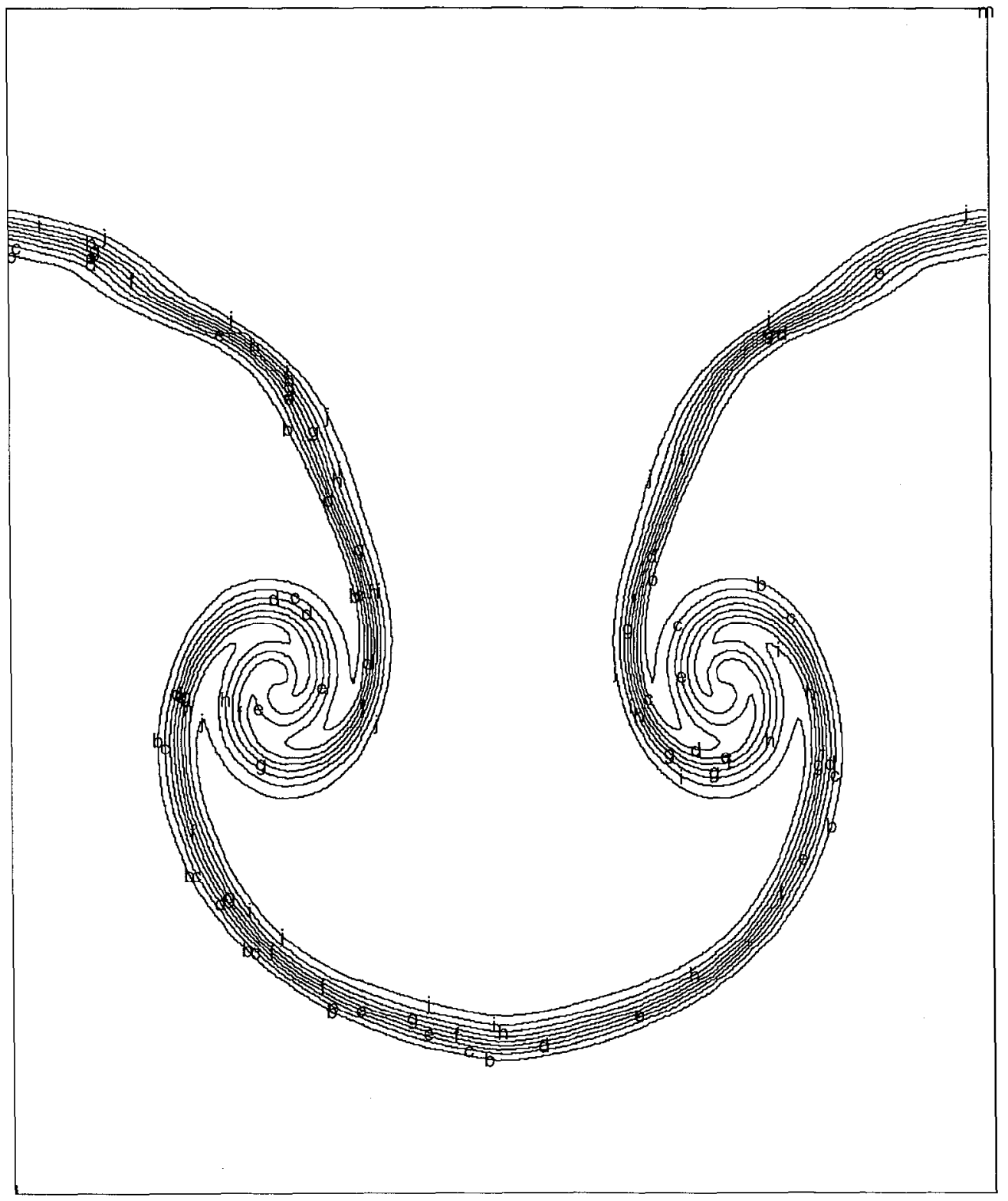

Figure 4: Mass fraction contours for species 1 at $40 \mathrm{~ms}$. The grid is $292 \times 352$, and the calculation is done with donor cell differencing and no turbulence model. 


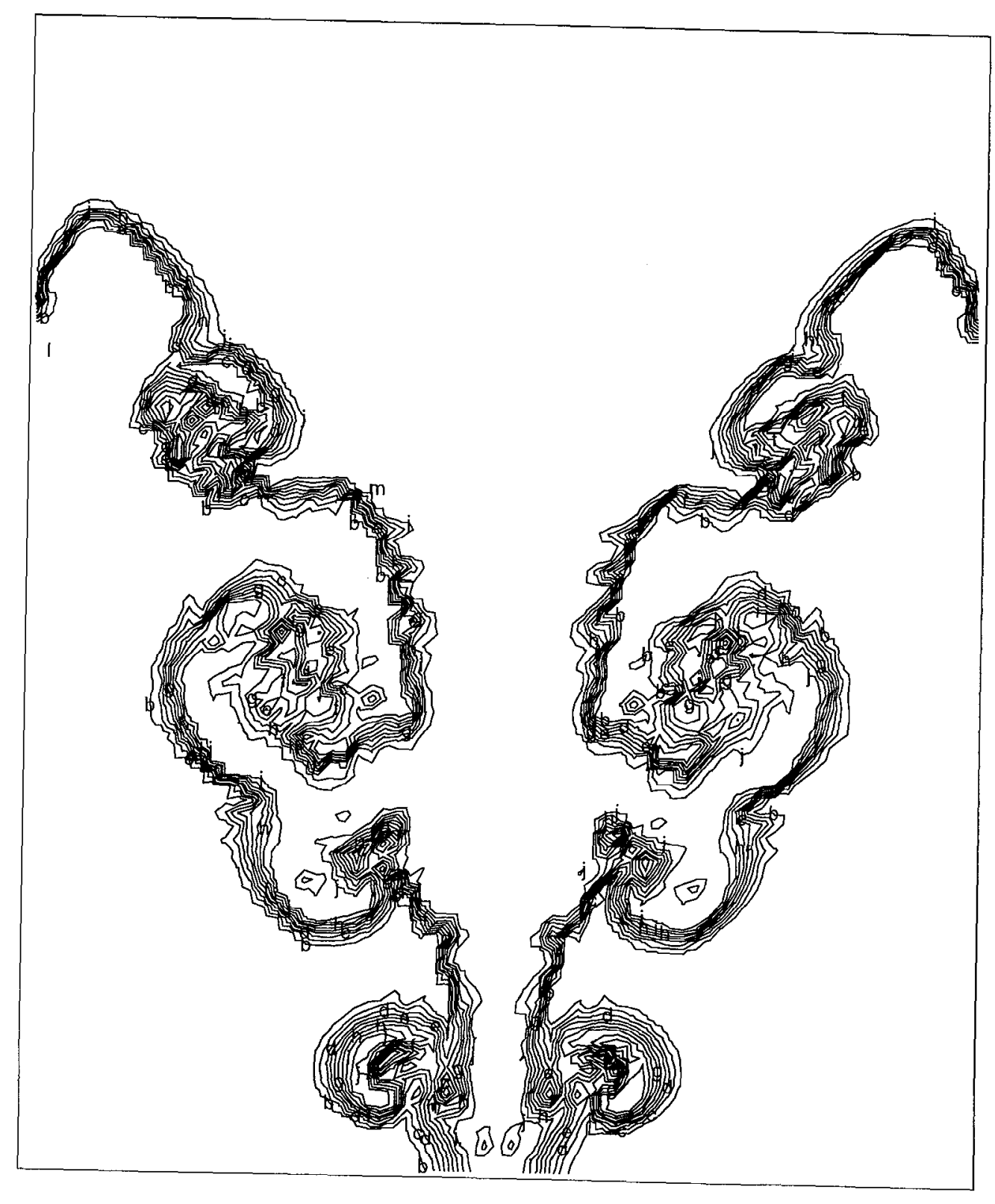

Figure 5: Mass fraction contours for species 1 at $40 \mathrm{~ms}$. The grid is $73 \times 88$, and the calculation is done with tensor viscosity differencing and no turbulence model. 


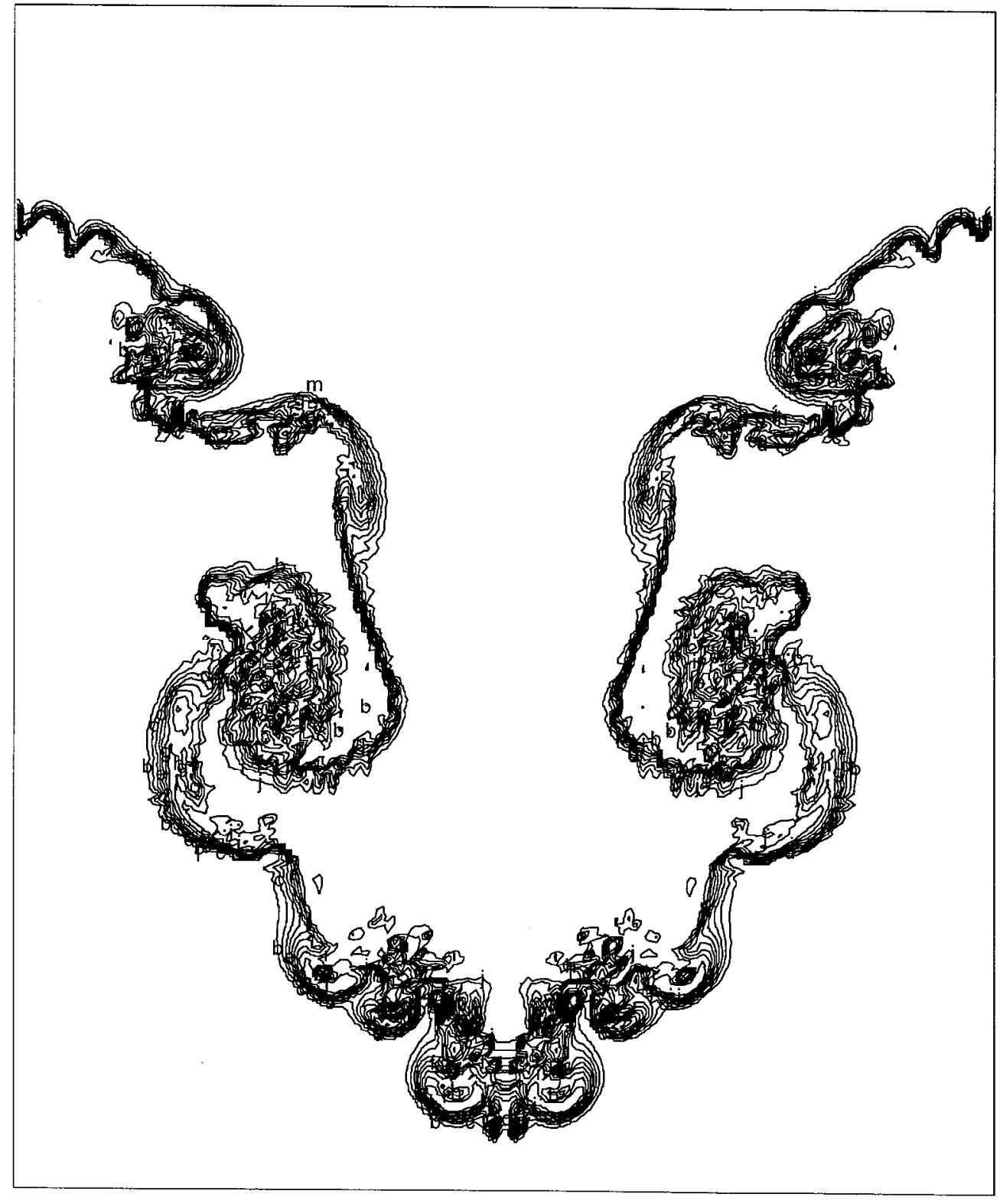

Figure 6: Mass fraction contours for species 1 at $40 \mathrm{~ms}$. The grid is $146 \times 196$, and the calculation is done with tensor viscosity differencing and no turbulence model. 


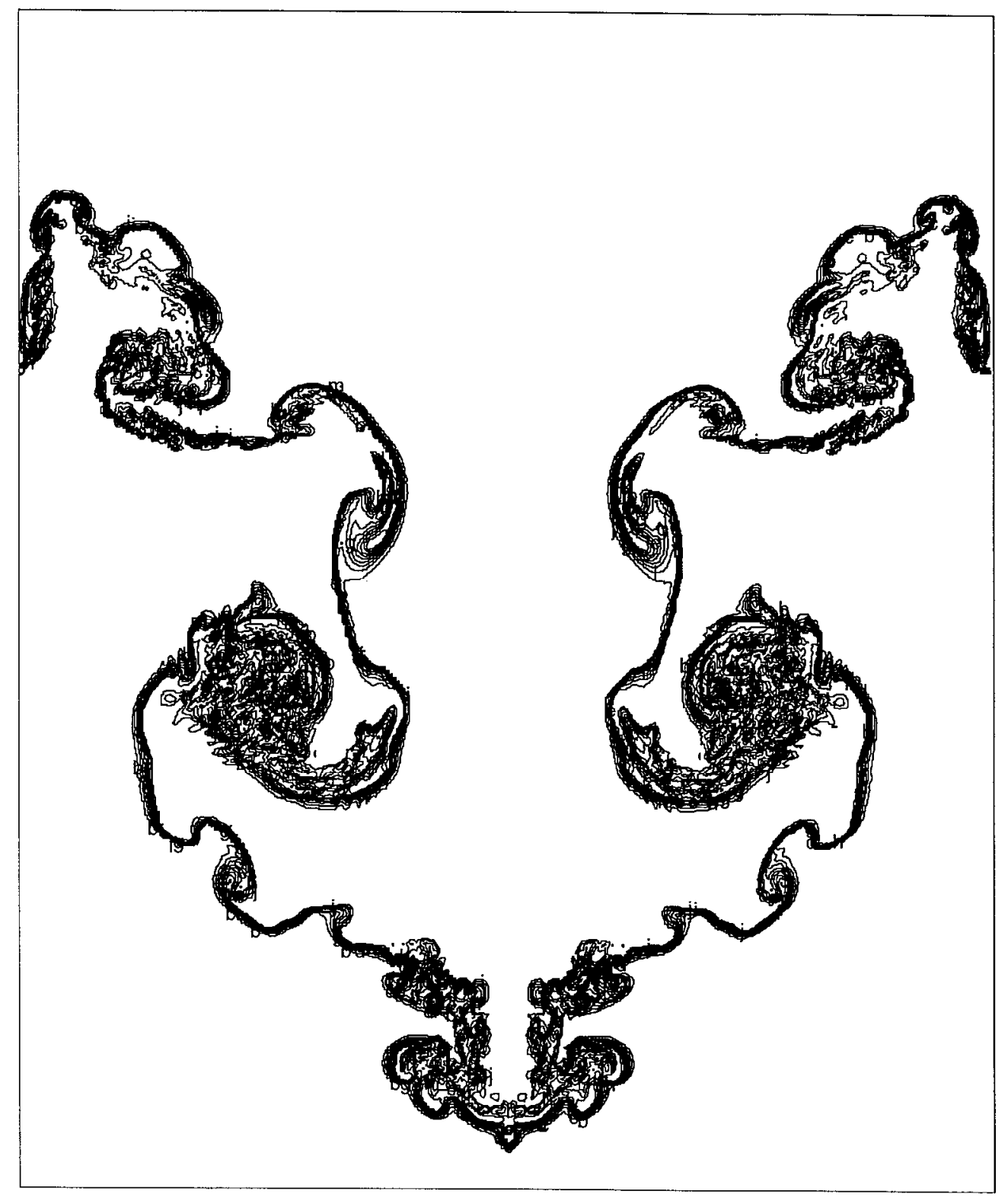

Figure 7: Mass fraction contours for species 1 at $40 \mathrm{~ms}$. The grid is $292 \times 352$, and the calculation is done with tensor viscosity differencing and no turbulence model. 


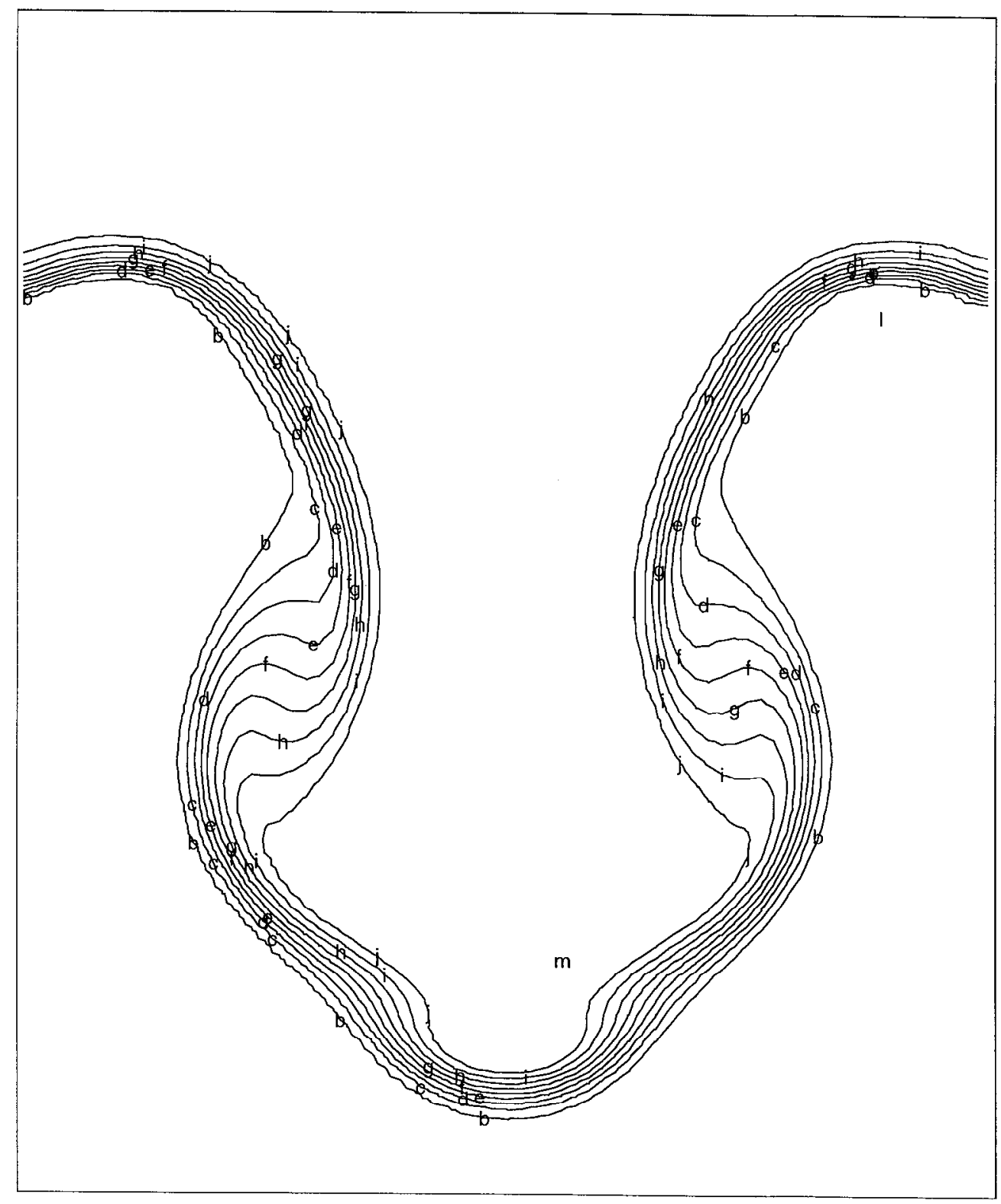

Figure 8: Mass fraction contours for species 1 at $40 \mathrm{~ms}$. The grid is $73 \times 88$, and the Smagorinsky model is used. 


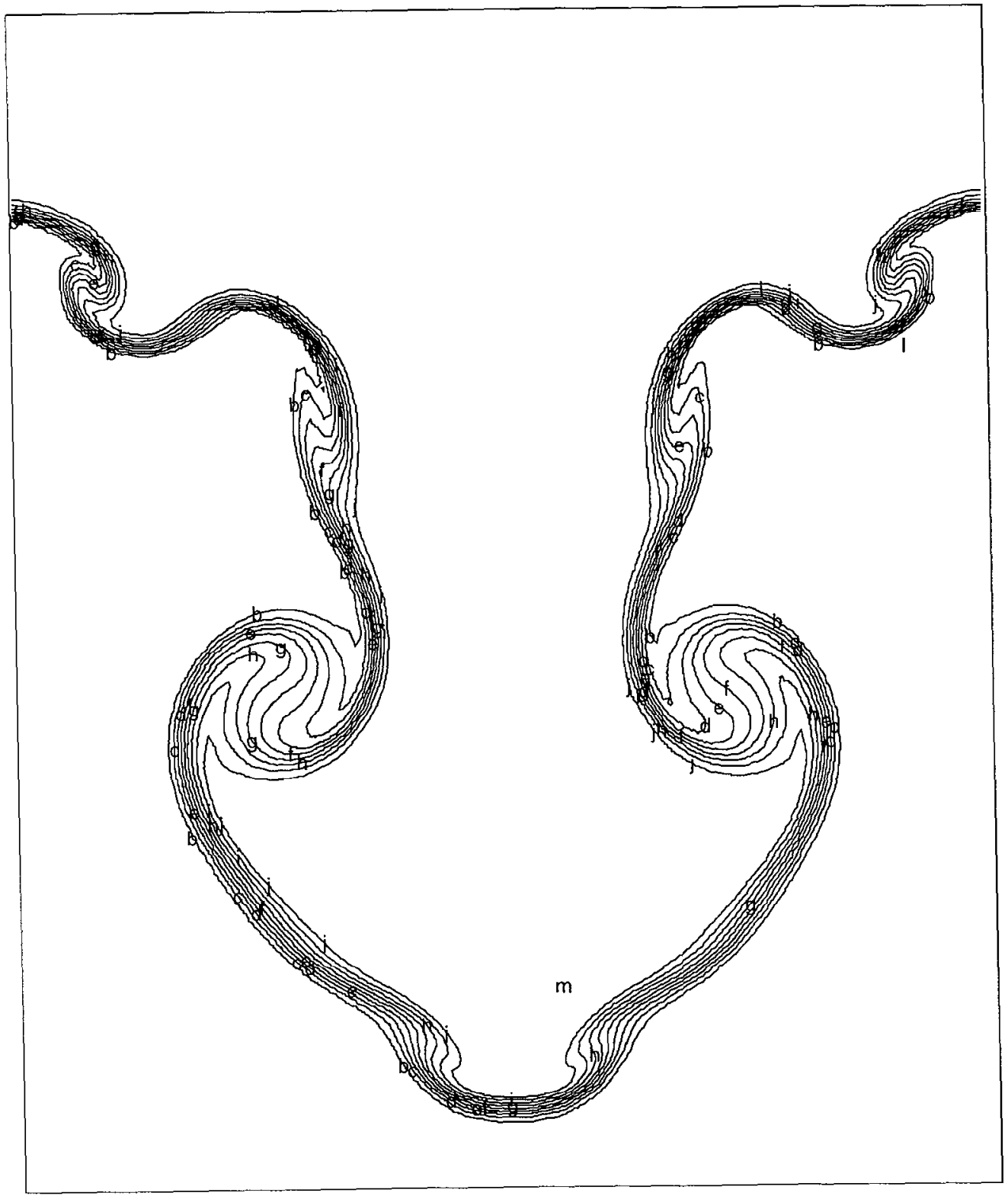

Figure 9: Mass fraction contours for species 1 at $40 \mathrm{~ms}$. The grid is $146 \times 196$, and the Smagorinsky model is used. 


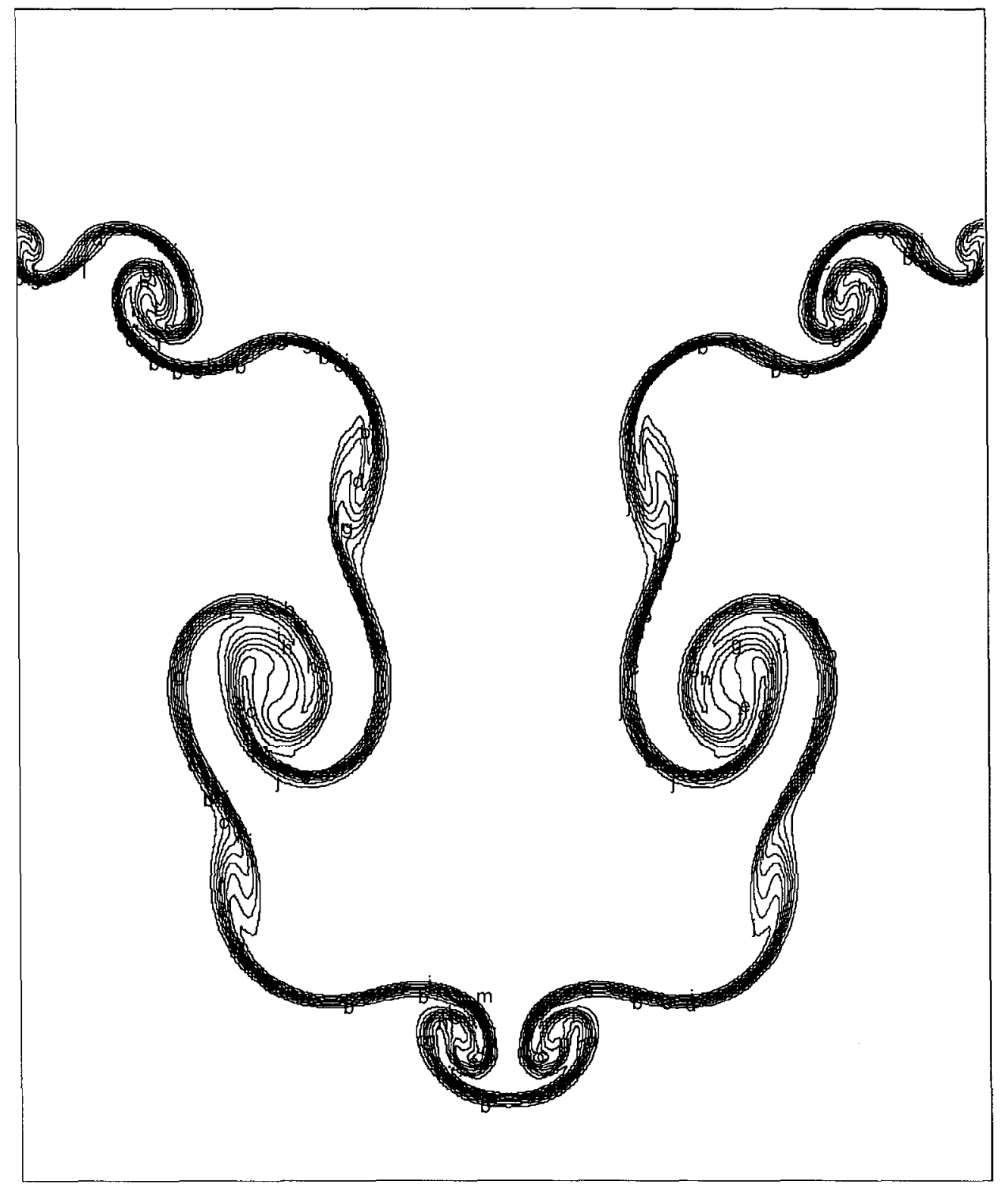

Figure 10: Mass fraction contours for species 1 at $40 \mathrm{~ms}$. The grid is $292 \times 352$, and the Smagorinsky model is used. 


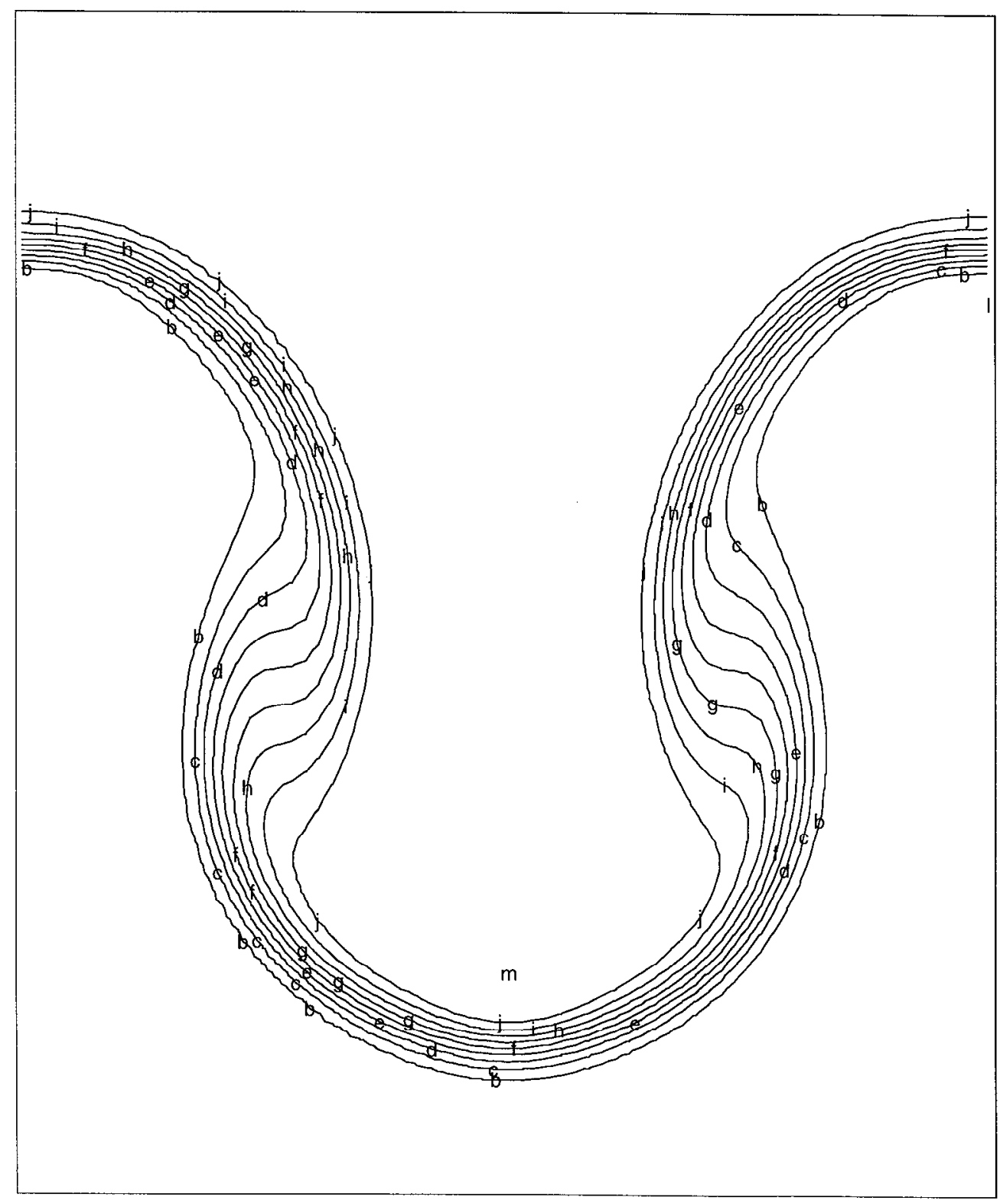

Figure 11: Mass fraction contours for species 1 at $40 \mathrm{~ms}$. The grid is $73 \times 88$, and the LUVD11 model is used. 


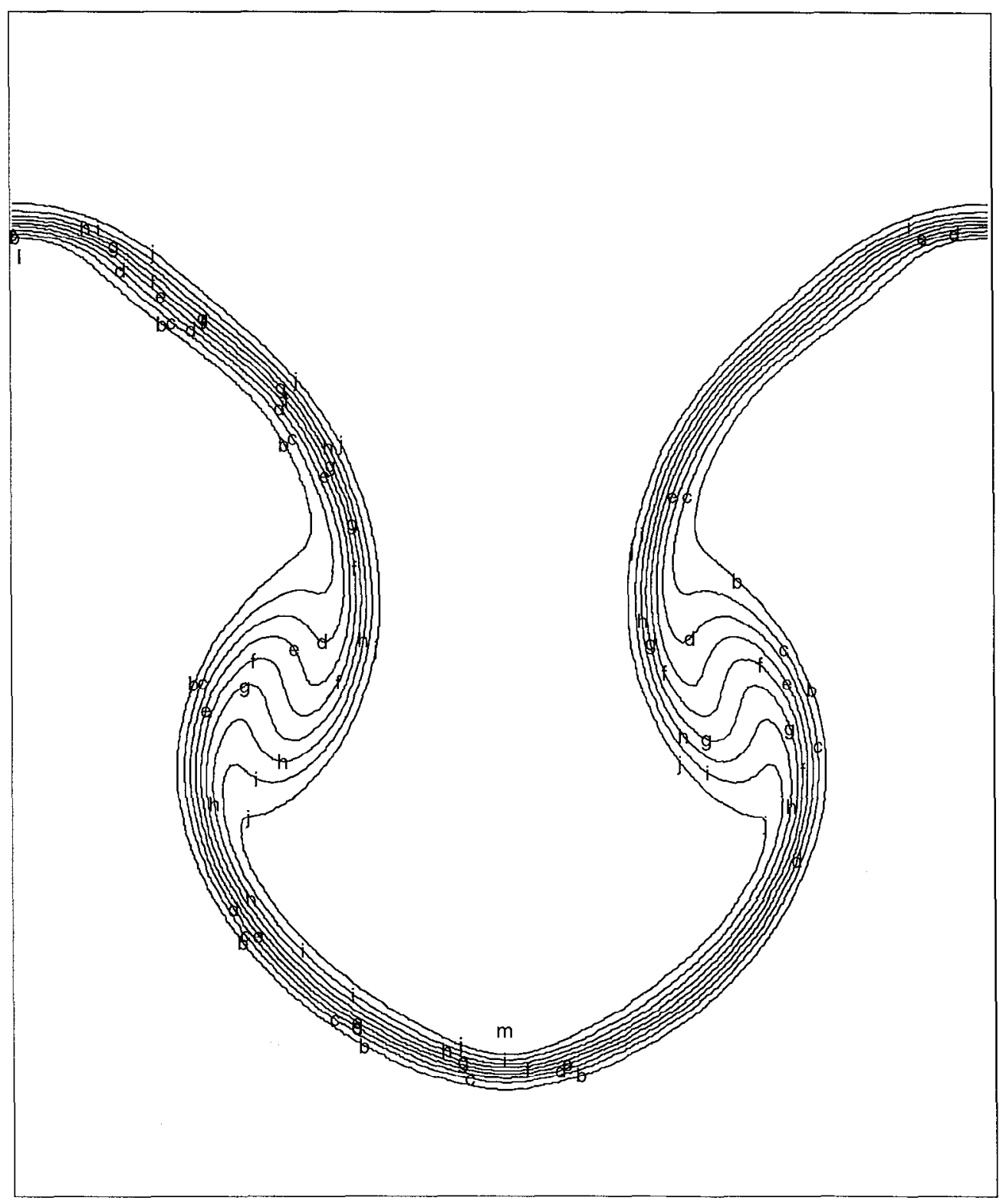

Figure 12: Mass fraction contours for species 1 at $40 \mathrm{~ms}$. The grid is $146 \times 196$, and the LUVD11 model is used. 


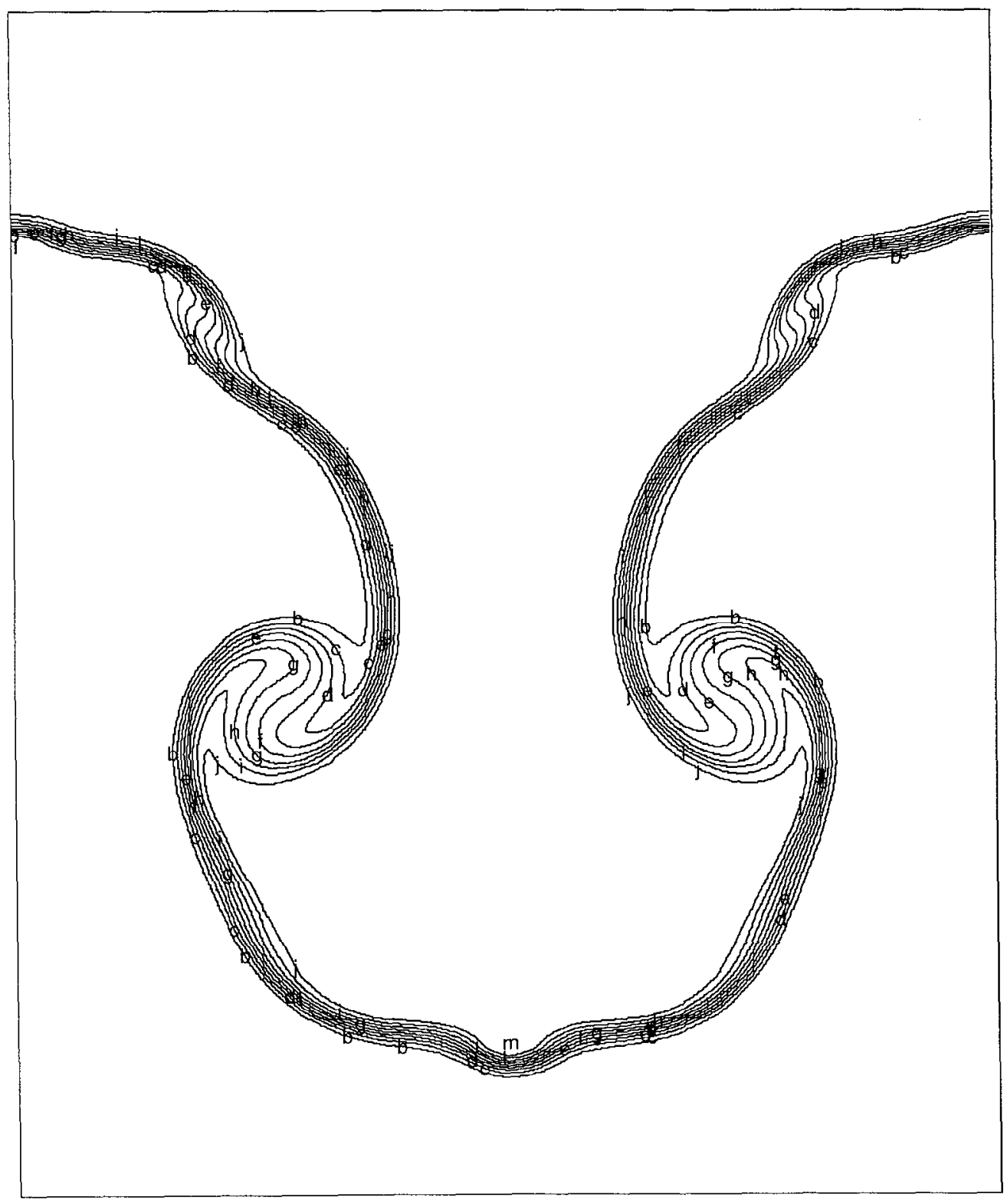

Figure 13: Mass fraction contours for species 1 at $40 \mathrm{~ms}$. The grid is $292 \times 352$, and the LUVD11 model is used. 


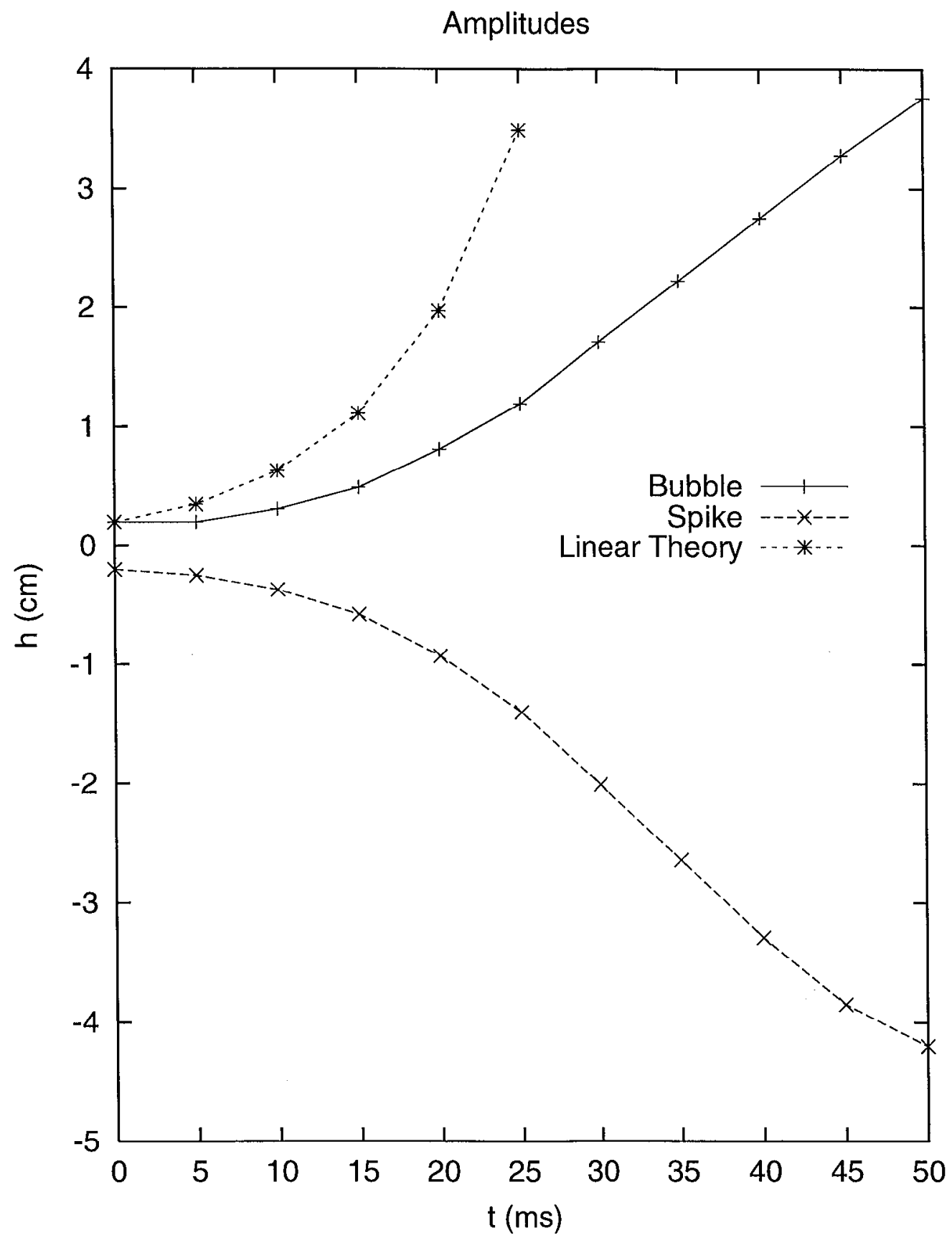

Figure 14: Bubble and spike amplitudes as a functions of time for the finest resolution donor cell run, figure 4. 


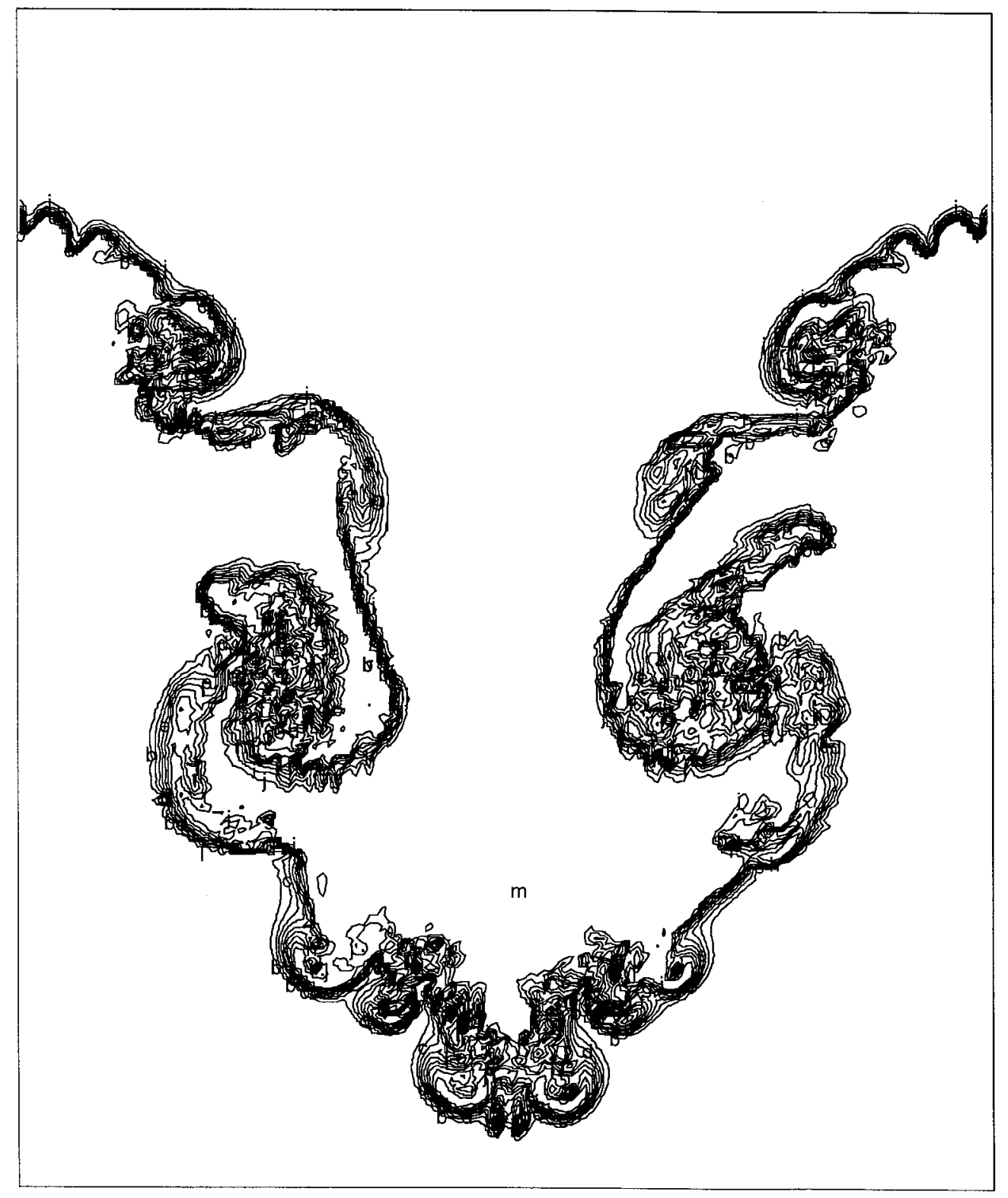

Figure 15: Mass fraction contours for species 1 at $40 \mathrm{~ms}$. The grid is $146 \times 196$, and the tensor viscosity method with no turbulence model is used. The initial condition was perturbed slightly to break the symmetry about the midplane, as shown in figure 1. Compare this plot to figure 6 . 


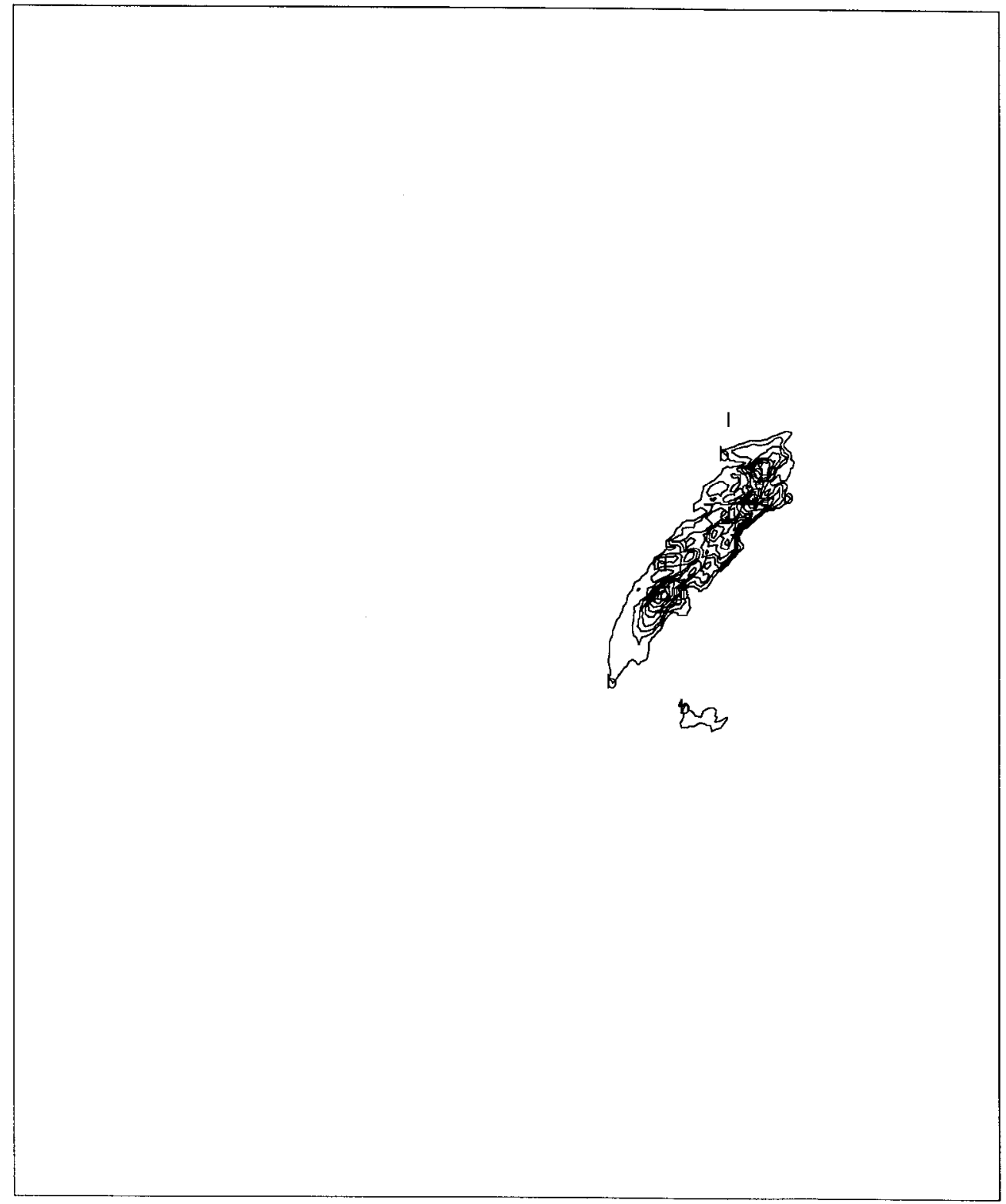

Figure 16: Mass fraction contours for species 3 at $40 \mathrm{~ms}$. The grid is $146 \times 196$, and the tensor viscosity method with no turbulence model is used. This is the small blob of fluid used to break the symmetry of the initial condition in figure 1, and the maximum mass fraction is 0.048 . 


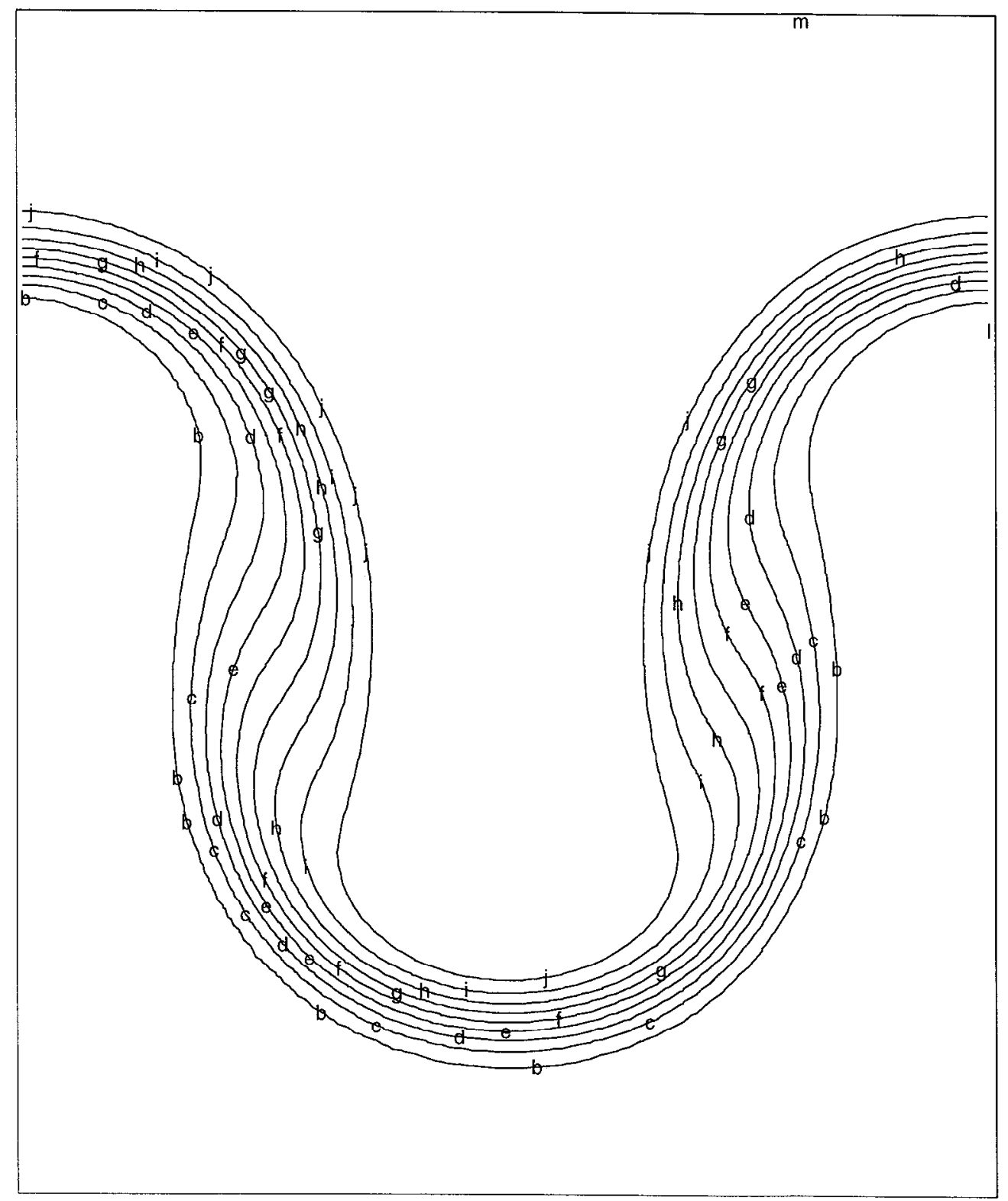

Figure 17: Mass fraction contours for species 1 at $40 \mathrm{~ms}$. The grid is $73 \times 88$, and the LUVD11 model is used with $L=3.75 \delta x$. 


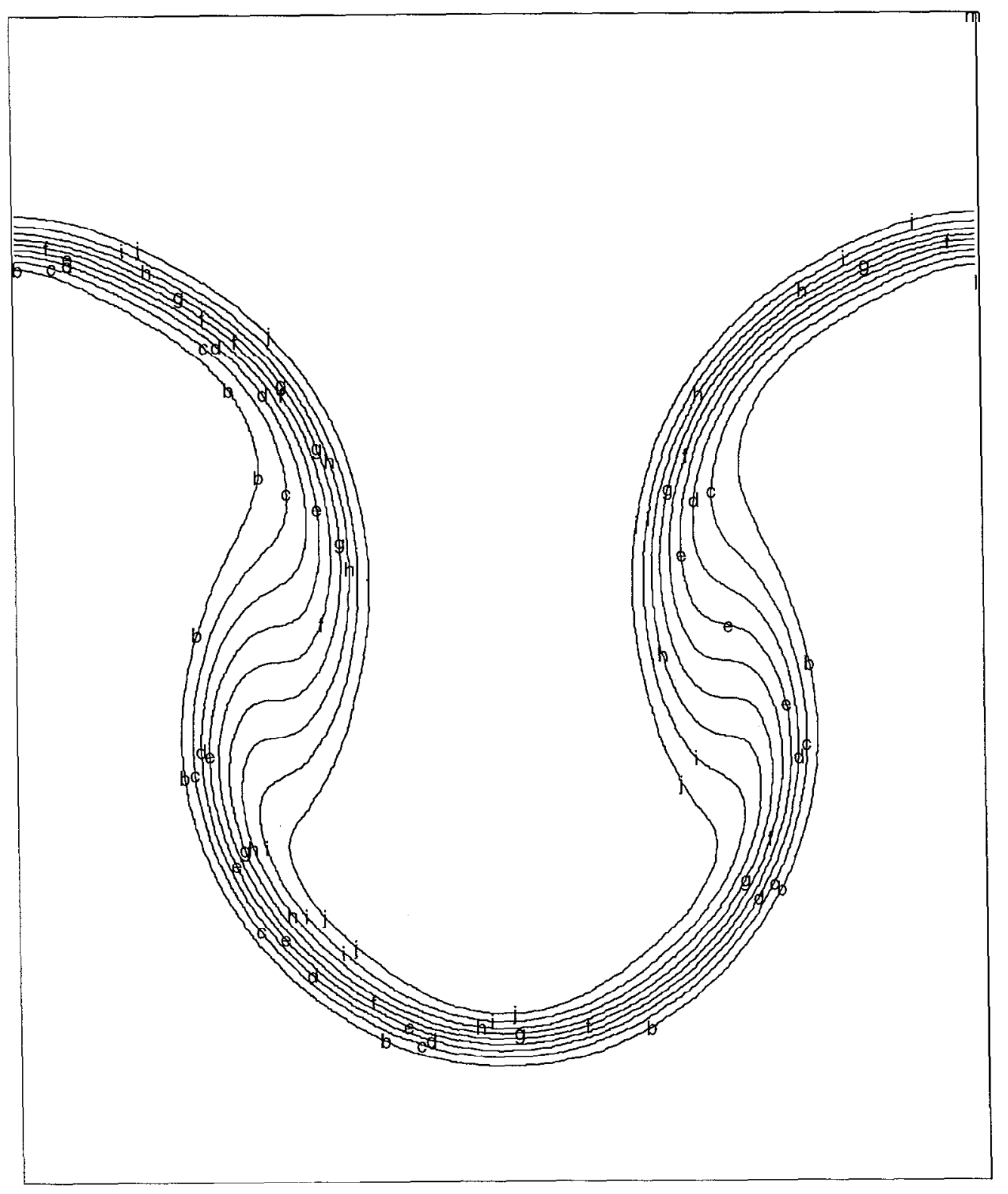

Figure 18: Mass fraction contours for species 1 at $40 \mathrm{~ms}$. The grid is $146 \times 196$, and the LUVD11 model is used with $L=3.75 \delta x$. 


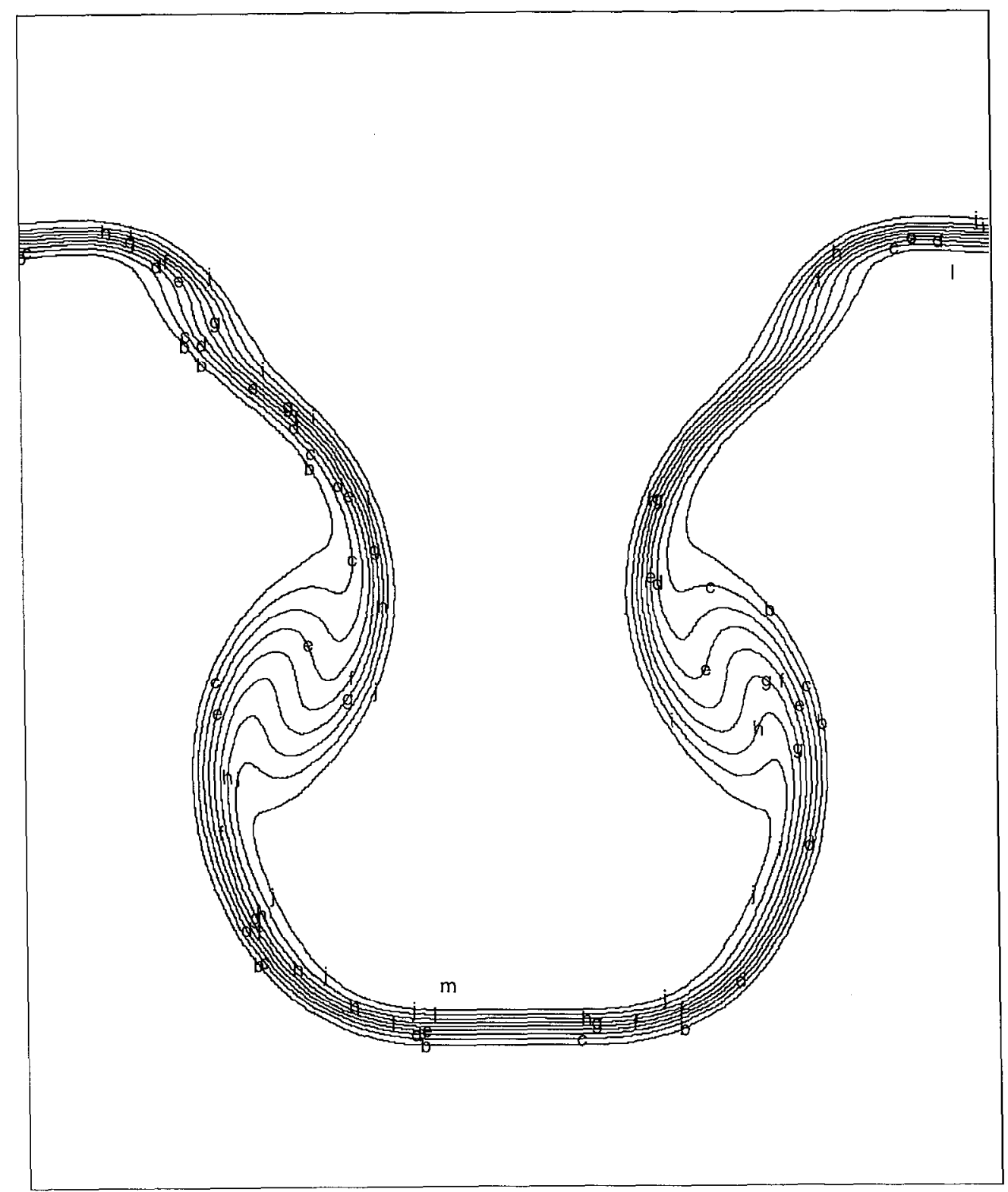

Figure 19: Mass fraction contours for species 1 at $40 \mathrm{~ms}$. The grid is $292 \times 352$, and the LUVD11 model is used with $L=3.75 \delta x$. 


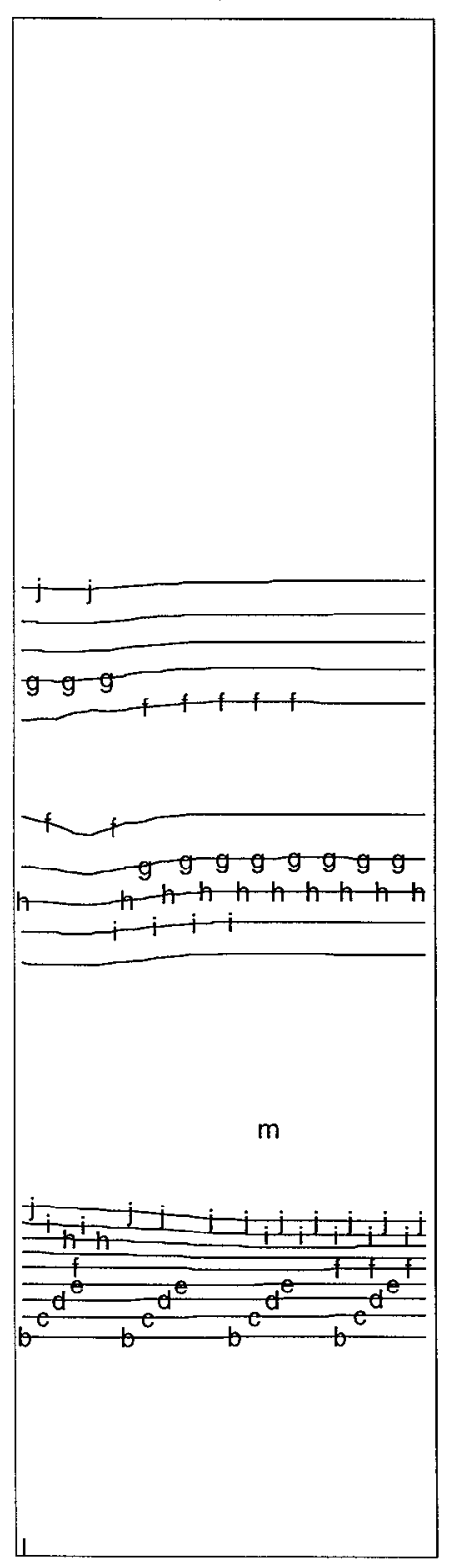

Figure 20: Mass fraction contours for species 1 at $40 \mathrm{~ms}$. The grid is $292 \times 352$, and the LUVD11 model is used with $L=3.75 \delta x$. 